Portland State University

PDXScholar

$10-9-2018$

\title{
Evolution from Cooling Modeling to Cooling Engineering of the Steel Quenching Process: A Technology Overview
}

Xinmin Luo

Jiangsu University of Science and Technology

George Totten

Portland State University, getotten@gmail.com

Follow this and additional works at: https://pdxscholar.library.pdx.edu/mengin_fac

Part of the Mechanical Engineering Commons

Let us know how access to this document benefits you.

\section{Citation Details}

Luo, X. and Totten, G. E., "Evolution from Cooling Modeling to Cooling Engineering of the Steel Quenching Process: A Technology Overview," Materials Performance and Characterization.

This Article is brought to you for free and open access. It has been accepted for inclusion in Mechanical and Materials Engineering Faculty Publications and Presentations by an authorized administrator of PDXScholar. Please contact us if we can make this document more accessible: pdxscholar@pdx.edu. 


\section{REVIEW PAPER}

Manuscript received February 7 , 2018; accepted for publication August 13, 2018; published online October 9, 2018.

1 School of Materials Science and Engineering, Jiangsu University, Zhenjiang, Jiangsu 212013, China (Corresponding author), e-mail: luoxm@ujs.edu.cn, (1) https:// orcid.org/0000-0003-1122-6956

2 School of Materials and Mechanical Engineering, Portland State University, Portland, OR 97207, USA, (1) https://orcid.org/ 0000-0002-7313-1437
Xinmin Luo ${ }^{1}$ and George E. Totten ${ }^{2}$

\section{Evolution from Cooling Modeling to Cooling Engineering of the Steel Quenching Process: A Technology Overview}

\section{Reference}

Luo, X. and Totten, G. E., "Evolution from Cooling Modeling to Cooling Engineering of the Steel Quenching Process: A Technology Overview," Materials Performance and Characterization https://doi.org/10.1520/MPC20180028. ISSN 2379-1365

\section{ABSTRACT}

The type of heat treatment process used is mainly determined by its cooling mode. Hardening is a metal heat treatment during which the most critical operation is cooling. Most quenchants used for steel hardening are vaporizable liquids, such as water, petroleum oils, emulsions, aqueous polymer solutions, or brines. Testing methodologies that include ISO 9950, Industrial Quenching Oils-determination of Cooling Characteristics-Nickel-alloy Probe Test Method, and ASTM Standards: D6200, Standard Test Method for Determination of Cooling Characteristics of Quench Oils by Cooling Curve Analysis, D6482, Standard Test Method for Determination of Cooling Characteristics of Aqueous Polymer Quenchants by Cooling Curve Analysis with Agitation (Tensi Method), D6549, Standard Test Method for Determination of Cooling Characteristics of Quenchants by Cooling Curve Analysis with Agitation (Drayton Unit), and D7646, Standard Test Method for Determination of Cooling Characteristics of Aqueous Polymer Quenchants for Aluminum Alloys by Cooling Curve Analysis, are typically used to evaluate the cooling characteristics of these quenchants or processes. The cooling timetemperature curves obtained by these methodologies traditionally involve three stages of cooling. However, the relationship between this model and heat treatment, especially between hardening theory and practice, has not been satisfactory. Therefore, it is necessary to better understand the relationship between cooling theory and metallurgical behavior to create a new cooling model that achieves zero distortion, zero dispersion of quality, and zero pollution, which 
are concepts proposed in the "Heat Treating Industry Vision 2020 "and the "Heat Treating Technology Roadmap 2004" by the ASM Heat Treating Society and Materials Treating Institute. The relationship between the physical metallurgical phenomenon of phase transformation of a steel workpiece during quenching after being austenitized and the actual cooling conditions in engineering practice were analyzed and discussed in this report. Additionally, the development of advanced cooling technologies and related quenching equipment are discussed. The evolution from cooling model research to cooling engineering construction represents important progress in heat treatment practice.

\section{Keywords}

steel heat treatment, hardening, distortion control, interface modeling, cooling medium, cooling engineering, quenching equipment

\section{Introduction}

The classical cooling model for vaporizable quenchants utilizes three stages of cooling. However, the relationship between this model and heat treatment, especially between hardening theory and practice, has not been satisfactory as will be explained. Therefore, it is necessary to better understand the relationship between cooling theory and metallurgical behavior to create a new cooling model to achieve the goals of "Zero distortion," "Zero dispersion of quality," and "Zero pollution" that have been proposed by the Heat Treating Industry Vision 2020 and the Heat Treating Technology Roadmap 200 initiated and issued by the ASM Heat Treating Society and Materials Treating Institute [1-4].

As for the cooling models, the first model is related to pure cooling. Originally, the cooling models for a vaporizable liquid coolant were established to account for the heat transfer that occurs when a hot solid object is immersed into a coolant that is typically characterized by three-stage cooling, which, however, is not related to steel hardening. The most important key issue relative to heat transfer upon steel quenching is the effect of the bulk coolant that surrounds the workpiece during quenching. Therefore, a three auxiliary interface model is suggested in addition to the main cooling interface models [5].

The second kind of model should be the steel hardening process itself. Traditionally, two hardening models, i.e., time-temperature-transformation (TTT) and continuous cooling transformation (CCT), account for the metallurgical phenomena for non-equilibrium processes and exclude the complicated and nonlinear factors that affect steel transformation upon quenching. Therefore, a series of relatively small test specimens is typically designed to avoid the volume effect. Unfortunately, there is no direct relationship between these models and hardening of the steel workpiece. Of course, distortion induced by stresses during quenching are not included in this model. Consequently, the actual hardening of a steel workpiece requires new models to guide heat treating practice.

Thus, a third kind of model for real heat treating practice should be developed for quench-hardening that establishes the concept of cooling engineering and connects the cooling process involved in steel hardening to the two inherently unrelated models to solve the issues of hardenability, distortion, and quenching efficiency of steel workpieces. Different cooling engineering to utilize the cooling characteristics based on the cooling 
model of vaporizable quenchants at different stages is discussed. The relationship between the physical metallurgical phenomenon of phase transformation of a steel workpiece during quenching after austenization and the actual cooling conditions in engineering practice was analyzed and will also be discussed here in addition to the importance of the development of advanced cooling technology and quenching equipment. Finally, included in this discussion, is the evolution from cooling model research to cooling engineering construction as it represents important progress in heat treatment practice.

\section{Discussion}

Heat treatment is one of the most important processes in steel manufacturing that involves changing the microstructure of the workpiece to provide the most suitable mechanical properties and service performance for as long as possible compared with other processing technology. Heat treatment should not change the workpiece geometry, designed size, or overall chemical composition. Quench processing is one of the most important components of the heat treatment operation.

Quenching may result in a completely different structure than what existing before heating in order to obtain a structure that produces optimal performance. With the development of the science and technology of quenching, the peculiar influence of the quenching agent on the overall quenching effect has been extensively explored. Since the 20th century, the development of materials science and engineering, together with the development and application of other new technologies, has resulted in notable progress in understanding the impact of quenchant media selection on the resulting material properties since the 1970s and 1980s, and some works are summarized in Refs. [6-21]. However, until now, the use of the research results of the heat treatment cooling process to systematically solve practical engineering problems is still in development. With the development of the International Federation for Heat Treatment and Surface Engineering (IFHTSE) and other national work on quench-cooling technology and distortion control technology, the heat treatment quench-cooling problem as a systematic engineering problem is now readily recognized.

Since the cooling process of quenching is so important, it is necessary to study the various factors associated with it; the most fundamental and most important aspect is the discussion of the research and cooling model of the cooling process as well as the cooling requirements for the optimal transformation of steel microstructure and incidental problems of the cooling process, including distortion control. This work is critical in view of the inevitable future automation of unmanned robot operations where cooling engineering and quenching automation are under current development.

\section{Measurement of Cooling Curves and Three-Phase Model}

Steel quenching media selection has been of interest since ancient times and is still studied today $[22,23]$. Thus, the study of quenching has long been known to begin with the cooling media.

The Quenching and Cooling (Q\&C) Committee of IFHTSE, which was chaired by Bozidar Liščić for many years, developed a standardized laboratory cooling curve analysis test that made it possible to effectively compare results obtained in different laboratories. 
In 1985, the Q\&C Committee developed the draft standard ISO/DIS9950, Determination of Industrial Quenching Oil-Cooling Characteristics-Laboratory Test Method, which subsequently became ISO 9950, Industrial Quenching Oils-determination of Cooling Characteristics-Nickel-Alloy Probe Test Method [24]. The draft standard was based on the Laboratory Test for Assessing the Cooling Characteristics of Industrial Media, published in 1982 by the Engineering Department's Quenching Media Group at the Wolfson Heat Treatment Center of University of Aston, Birmingham, United Kingdom. Subsequently, ASTM published an equivalent standard designated ASTM D6200, Standard Test Method for Determination of Cooling Characteristics of Quench Oils by Cooling Curve Analysis [25].

The ISO 9950 and ASTM D6200 standards both utilize 12.5 by $60-\mathrm{mm}$ cylindrical Inconel 600 probes with a Type $\mathrm{K}$ thermocouple inserted to the geometric center. Other ASTM cooling curve standards that were later developed that utilize the same 12.5 by 60-mm Inconel 600 probe include: ASTM D6482, Standard Test Method for Determination of Cooling Characteristics of Aqueous Polymer Quenchants by Cooling Curve Analysis with Agitation (Tensi Method) [26] and ASTM D6549, Standard Test Method for Determination of Cooling Characteristics of Quenchants by Cooling Curve Analysis with Agitation (Drayton Unit) [27]. Fig. 1a shows an ISO 9950 standard Inconel 600 probe. Fig. 1b shows the Chinese silver probe (SH/T 0220-1992, Heat Treatment Oil Cooling Characteristics Test Method), which is mainly used to test the cooling characteristics of industrial quenching oil and was later extended to test organic aqueous solution quenching media [28]. The introduction of ISO standard probes in China has made reference to the establishment of a new standard, that is, the laboratory method for determining the cooling characteristics of industrial quenching oils, JB/T 7951-2004, Industrial Quenching Oil. Determination of Cooling Characteristics. Nickel-Alloy Probe Test Method, a lab method that uses nickel alloy probes to test cooling characteristics of industrial quenching oil [29]. At present, the latter testing methodology is becoming more dominant.

The purpose of the probes illustrated in Fig. 1 is to obtain a standardized cooling curve of the quenching medium being measured. Fig. 2 shows a typical cooling

FIG. 1 (a) Diagrams of the Inconel 600 probe and (b) a silver probe for measuring cooling curves of quenchants.
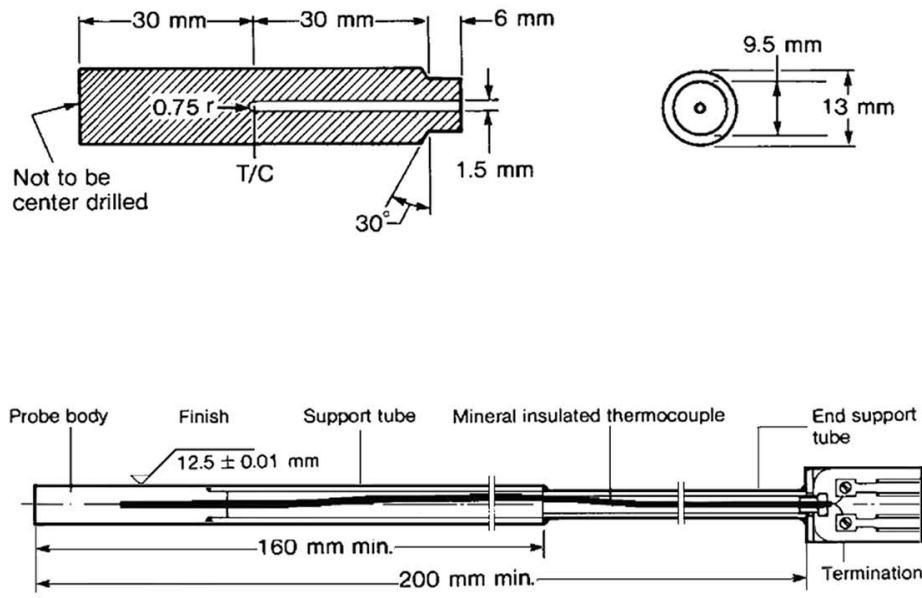

(a)
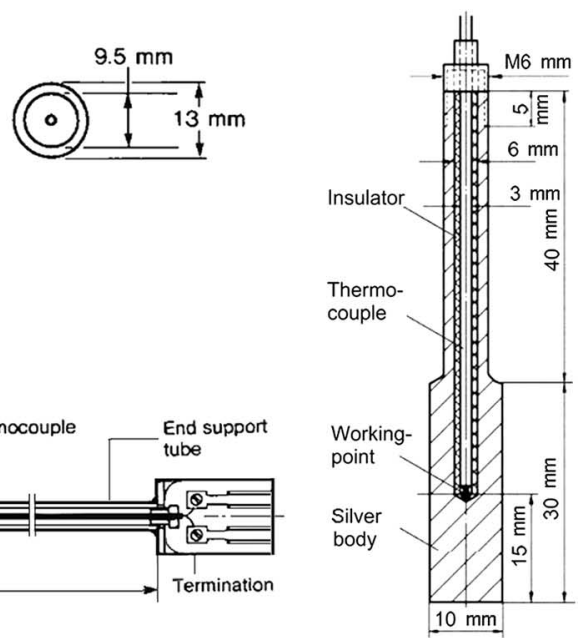

(b) 
FIG. 2 (a) Typical cooling time-temperature curve and (b) cooling-cooling rate curves using an Inconel 600 probe.

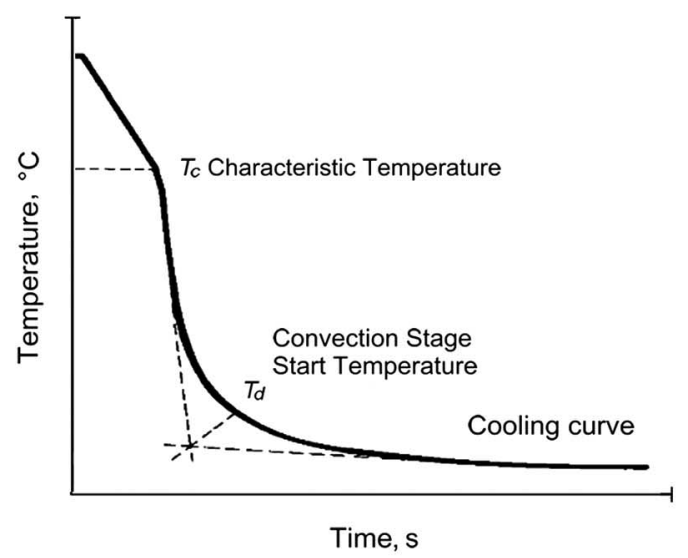

(a)

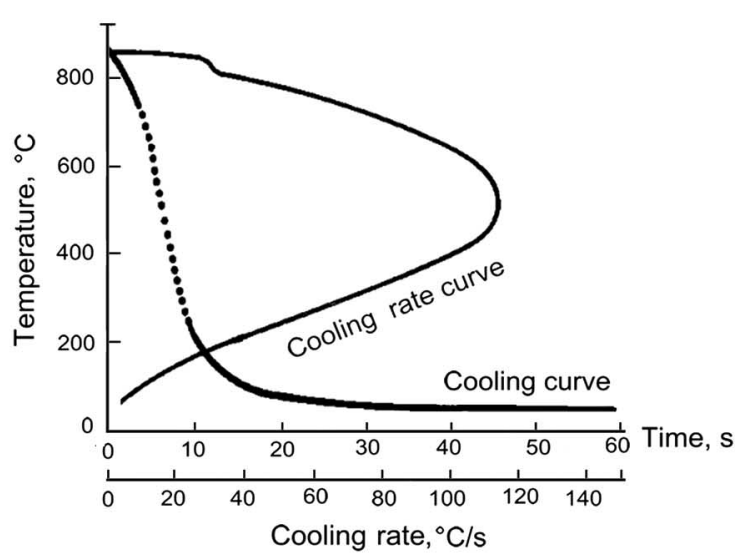

(b)

time-temperature curve and a cooling rate curve obtained for a vaporizable quenchant that is characterized by three characteristic cooling phases [30].

It is important to note that such cooling curves obtained under laboratory testing conditions do not necessarily relate to the actual heat transfer of the cooling process of an actual component in an industrial heat treating shop, though they do permit comparison of heat transfer characteristics under standard laboratory conditions. Essentially, these testing methods comparatively assess the physical heat transfer when a hot object without an internal heat source is being immersed into a vaporizable cooling medium. These laboratory testing procedures are useful for research, quenchant development, and quality control. However, they do not necessarily relate to actual quenching experience in the heat treating shop. For this reason, the currently most often encountered cooling model for vaporizable quenchants should be re-examined for its applicability (using small probe cooling curve data) for industrial quenching process understanding and design.

\section{Three-Phase Cooling Model and Main-Auxiliary Interface Model upon Surface Cooling}

\section{THE BASICS OF THE THREE-PHASE MODEL}

Fig. 3 shows the phenomenon observed in the immersion of a hot object into a vaporizable cooling medium, as shown in this figure: a cooling curve and a cooling rate curve [30]. Obviously, the cooling curve can be divided into three different heat transfer stages, namely, Stage A, vapor blanket cooling (film boiling) phase; Stage B, nuclear boiling cooling stage, and Stage $\mathrm{C}$, convective cooling phase.

As shown in Fig. 3, Stage A indicated on the curve is termed the vapor blanket cooling stage. During this first stage, upon initial immersion of the hot metal into the vaporizable liquid quenchant, a certain amount of liquid that is adjacent to the metal surface vaporizes and forms a continuous film or blanket of vapor around the cooling metal. Cooling proceeds through this film mainly by radiation and, consequently, a slow cooling rate is the result. 
FIG. 3

Cooling mechanisms.

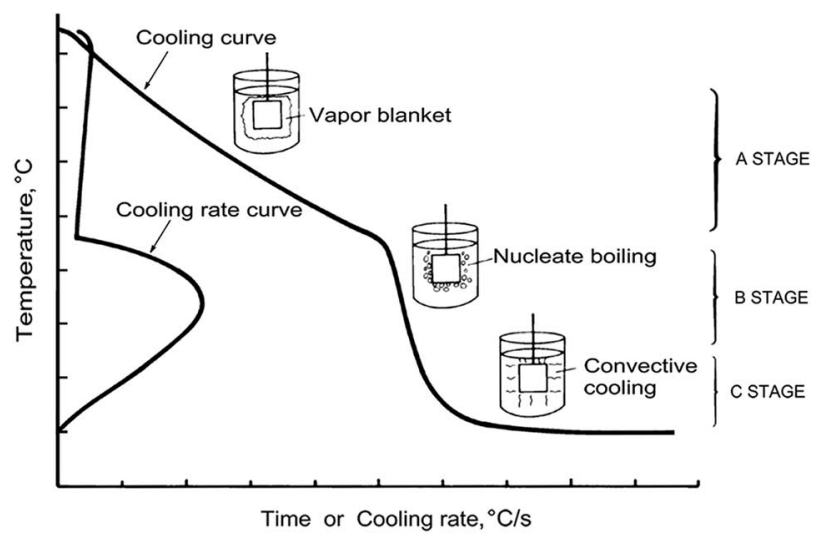

The second stage of cooling, Stage B, as indicated on the curve in Fig. 3, is the nucleate (violent) boiling stage where the continuity of the vapor blanket is lost. A certain amount of the vapor migrates into the liquid and condenses; new liquid rushes in and vapors at the hot object surface extract an amount of heat equal to the heat of vaporization of the liquid and is subsequently condensed in the body of the quenchant. This action is typically relatively violent and results in a rapid extraction of heat from the cooling surface at a rate largely dependent on the amount of liquid material that is vaporized.

When the temperature of the surface of the hot object becomes lower than the boiling point of the liquid, vapor can no longer form, and the second stage of cooling is completed. Cooling from this point on is designated Stage C, as indicated on Fig. 3, and occurs by conduction and convection. This cooling process produces a much slower rate of cooling than the preceding nucleate boiling stage. The rate of cooling here depends largely on the specific heat of the quenchant, its thermal conductivity, and the degree of agitation of the quenching bath.

There are many different opinions on the interpretation of the cooling curve for vaporizable cooling medium with phase changes, but these three phases can be considered to be the most relevant, and they represent the classical theoretical interpretation of the quenching process [31].

\section{ESTABLISHMENT OF MAIN-AUXILIARY INTERFACE MODEL}

Upon immersion, these three phases are actually in a closed cooling environment surrounded by a body of cool media at the cooling interface. Therefore, the three-phase heat transfer coefficient is extremely difficult to calculate. Today, the various methods of calculating the heat transfer coefficient are debated continuously, and in the meantime, even the results obtained are still difficult to apply in heat treatment.

Analysis of the cooling mode occurring on the steel surface upon immersion quenching will now be addressed with the objective of modification of the cooling model. Fig. 4 shows the surface cooling interface model based on the three-phase theory when a hot steel is immersion-quenched into a vaporizable quenchant in a stationary state.

According to the main-auxiliary interface model of the cooling interface shown in Fig. 4, it can be seen that when hot (austenitized) steel is immersion-quenched into a 
FIG. 4 The main-auxiliary interface model based on a three-stage model upon quenching.

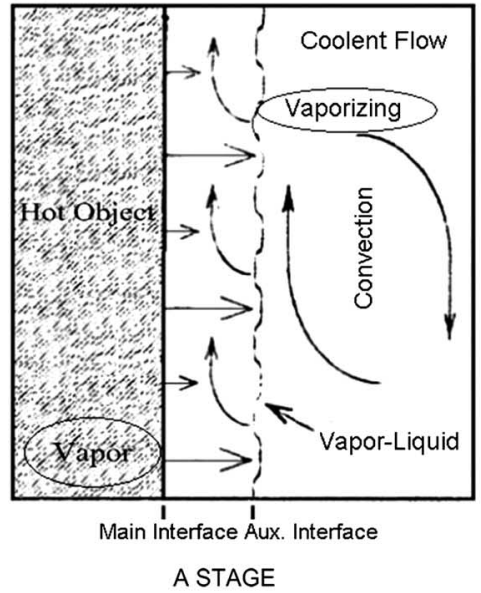

(a)

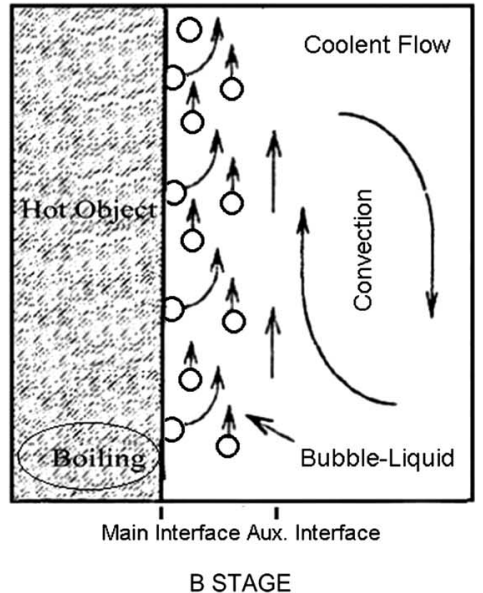

(b)

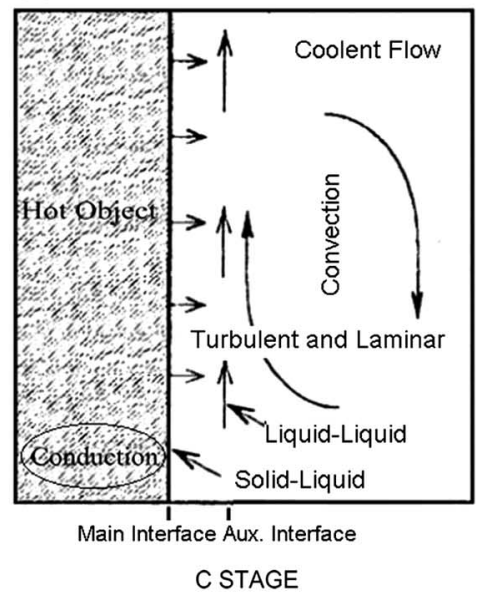

(c)

cooling medium, which is with a phase change, surface cooling is actually separated from the quenching medium itself as a result of vapor phase formation. Thus, the cooling front is formed just on the surface of the steel, i.e., the main cooling interface forms between solid surface and vapor phase. Because the vapor is fairly steady, which is due to the internal heat source effect of a hot workpiece, a second heat transfer interface is formed between the vapor phase and the quenchant, which is termed the auxiliary interface. Remarkably, the vapor on the main interface surrounded by the outside quenchant is not so free as in open conditions, which becomes the main feature of so-called pool cooling, the confinement effect of the surrounding media cannot be neglected. From this model, the role and the mechanism of the main and that of the auxiliary heat transfer interface are completely different.

In Stage A of the vapor blanket cooling, the main interface is a solid-vapor interface but the auxiliary interface is a vapor-liquid interface. From the main and auxiliary interface of the cooling diagram, the main interface is vapor-solid cooling, the essence of which is gas phase cooling within the bulk liquid. The surrounding gas layer is not static and should have all of the cooling characteristics of a single gas-phase fluid, but its pressure is affected by the surrounding cooling media and the immersion depth of the steel in the quenching tank. Although the boundary of the auxiliary interface (vapor-liquid interface) does exist and can be observed experimentally, the calculation method is not very clear. However, the dominant heat transfer process during Stage A cooling is at the steel surface, and the main role of the cooling medium is that it is the source of the vapor. Notably, this is very different from the pure vapor phase cooling in an open system since the cooling capacity is much lower.

During Stage B of nuclear boiling, the main interface is a solid-bubble interface, but the auxiliary interface is the bubble-liquid interface. The vapor-liquid interface in Fig. 4a is now destroyed, as shown in the main-auxiliary interface in the cooling diagram. The cooling main interface evolved into a vapor and liquid dual-phase flow cooling mode, but in essence, it is gas-liquid dual-phase flow cooling when surrounded by the bulk liquid. 
At this time, the auxiliary interface becomes difficult to determine, so the calculation method will be discussed subsequently.

In Stage $\mathrm{C}$ of convective cooling, the main interface is a solid-liquid interface, but the auxiliary interface is a turbulent flow-laminar flow interface. From the diagram, it appears that the auxiliary interface nearly disappears. But, in fact, there is a turbulentlaminar interface. However, at this time, the main interface completely transitions into single-phase (liquid phase) cooling, which is the actual situation during "immersion-bath" cooling in production quenching tanks. However, because of the viscous characteristics of a body of fluid, the effect of the boundary layer on cooling must be considered at this stage so the influence of various hydrodynamic factors on the cooling effect cannot be neglected, and the importance of stirring and its control on cooling uniformity becomes substantial.

\section{REVELATION OF THE MAIN-AUXILIARY INTERFACE MODEL}

The main-auxiliary interface model shows that the three-stage model is independent but not free and open and it is also largely influenced by the external viscous cold fluid and the gravitational pressure head. The cold fluid that surrounds the main cooling interface is not directly involved in the cooling for all three stages but instead limits the full interaction of the cooling capacity of each characteristic stage of the three-phase theory, Therefore, the three-stage theory of immersion quenching must be liberated from the outer regions in order to thoroughly understand immersion quenching.

In view of the main-auxiliary interface model based on the three-stage theory of the cooling process, the cooling media or cooling methods that exhibit one cooling stage can be conveniently controlled by manual intervention. Moreover, no matter what stage, only when solving the constraints of the surrounding fluid can the cooling model truly reflect the cooling characteristics of the quenchant or cooling method.

It is understood that the fewer the cooling phases involved in the entire cooling process, the better the uniformity and stability of the cooling process. However, there are still many factors that influence the quenching process. For example, the physical properties of the medium, which include density, specific heat, thermal conductivity, etc., will directly affect the heat transfer coefficient; temperature difference of the cooling couple, together with the latent heat of the steel part during phase transformation, will affect the kinetic process. Other factors include the condition of the surface of the part to be quenched, such as heat transfer area, surface cleanness, and geometrical shape, will influence the heat transfer. The static/kinetic factors of the cooling fluid itself, such as concentration, viscosity, temperature, pressure, flow rate, flow volume, angle, flow pattern, and boundary layer, also affect the heat transfer efficiency.

Based on the new concept of cooling uniformity requirements, why should the process with only one cooling stage be the easiest to adjust or control? The essence of the cooling process lies in Newtonian cooling-a linear cooling process that is relatively easily regulated to adjust cooling speed. However, it should be noted that at this time, even if the cooling is stable, the cooling rate is still constantly changing with the temperature difference of the cooling couple. Instead, it is more stable and is mainly controlled by the temperature difference and the physical properties of the cooling medium. Thus, a series of single-phase cooling modes can be derived, such as convection cooling, i.e., gas quenching, lead bath cooling, nitrate salt bath, and fluidized bed, or direct rupture of the surrounding fluid region, using such a process as spray cooling, so that cooling is intermediate between the boiling phase and convection phase. 


\section{Concept Cooling Models and Actual Quench-Cooling}

The cooling models that are closely related to the physical metallurgical phenomena of steels are TTT diagram and CCT diagram. The most important control factor of TTT diagram is a series of supercooled degrees between $A_{1}$ and the fixed temperature upon transformation; the most important control factor of the CCT diagram is a series of preset constant cooling rates. The common denominator of the two models is that the cooling method determines the phase transformation process of steel, and the other is that this process is totally unrelated to the three-stage cooling model.

It is known that the microstructure of materials may have special properties when far from the thermodynamic equilibrium state. The essence of heat treatment is to study various metallurgical phenomena of an alloy system deviating from the equilibrium state when the thermodynamic environment changes and cooling is the most commonly used method to affect the deviation. In this sense, both TTT and CCT are physical metallurgical cooling models.

The main objective of heat treatment of steel is that when the iron-carbon alloy system is heated, the homogeneous austenitic structure, which depends on the diffusion process, will form in a process of energy accumulation. But phase transformation upon quenching will deviate greatly, depending on the extent from the equilibrium of the system, i.e., the cooling intensity.

Therefore, if the quenching process is to obtain a nonequilibrium microstructure during an instantaneous process, it must make full use of the energy of the alloy system to meet the mutual adaptation between structure and energy. In this process, cooling becomes an issue of system engineering. At this time, in addition to the microstructure evolution within the alloy system, the thermodynamic and kinetic problems of the steel component to be cooled are highlighted as the main contradiction restricting the entire process. Therefore, the consistency between phase transition (physical metallurgy) and cooling kinetics (engineering) must be solved.

Fig. 5 provides a schematic diagram of the establishment of TTT and CCT diagrams. Both TTT and CCT diagrams reveal the isothermal transition kinetics and continuous cooling kinetics of austenite, respectively, which becomes the most important guide for designing the heat treatment process of steels. However, test results of the quenching medium's cooling characteristics lead to another issue, that is, almost no cooling speed mode or distribution of an actual workpiece in the entire process of quenching is consistent with the conditions of the cooling rate that was used to establish the TTT/CCT diagram.

It can be seen from Fig. 5a that the transformation of a steel specimen in the TTT diagram is an isothermal process depending on $\Delta t$, the temperature difference between $\mathrm{A}_{1}$ and the transformation temperature for target products. If now the latent heat is neglected, the cooling rate upon transforming should be zero; so far, there is no information or regulation that can be used on the determination of the cooling rates before the specimens reach the designed isothermal cooling temperatures. In the CCT diagram, the transformation process of the steel specimen after being austenitized is a continuous cooling transformation process where each cooling curve in the diagram is predetermined using constant cooling transformation speeds and recording only the starting and ending points of the phase change and resulting hardness. Because the types of the cooling 
FIG. 5 Cooling curves in (a) TTT diagram and (b) CCT diagram.

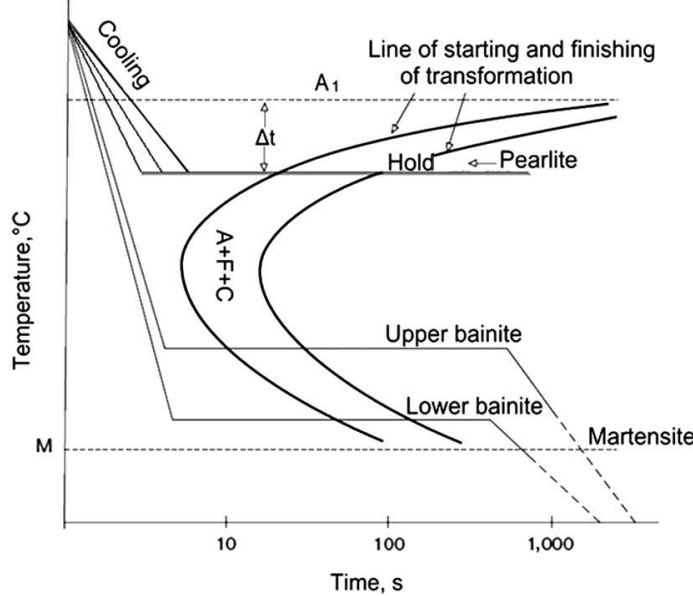

(a)

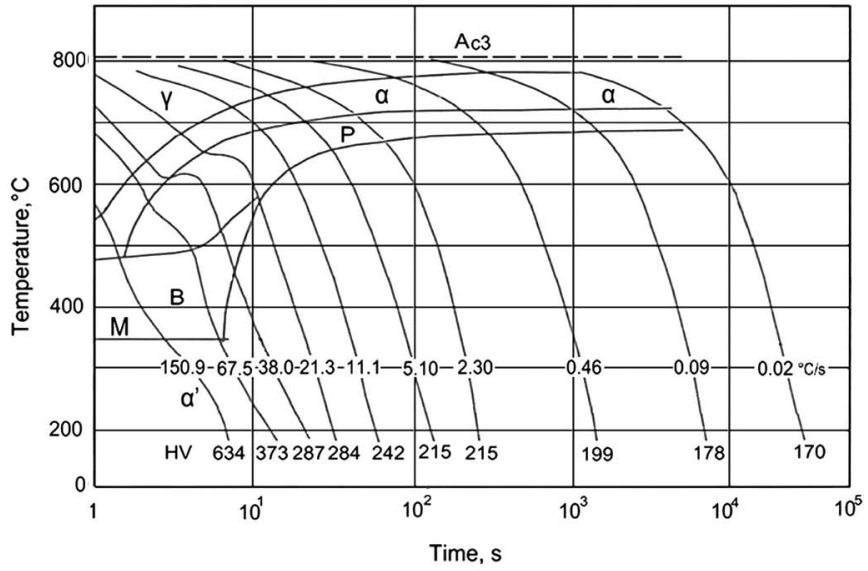

(b)

transformation processes of austenite is set to conduct the physical metallurgical study of steels, all of the internal and external interference factors are shielded in order to extract the characterization method, which is difficult to achieve in the actual quenching operation of steel parts. Therefore, they should be called "cooling concepts."

During an actual industrial immersion quench-cooling process, workpieces are not cooled as modeled by either TTT or CCT processes. Fig. 6 shows cylindrical probes that measure the cooling rate change of a steel workpiece when immersed in a vaporizable quenching medium. Fig. 6 a shows a laboratory cooling model of a vaporizable quenchant. It can be seen that the cooling rate of an actual object during quenching changes within a large range of decreasing temperatures, and the surface temperature of a workpiece fluctuates greatly as shown in Fig. $6 \mathrm{~b}$. Thus, such a cooling characteristic curve cannot represent the cooling rate for any actual steel part upon quenching. Therefore, the cooling

FIG. 6 (a) Cooling characteristic variation with temperature and (b) surface temperature fluctuation of a workpiece upon cooling.

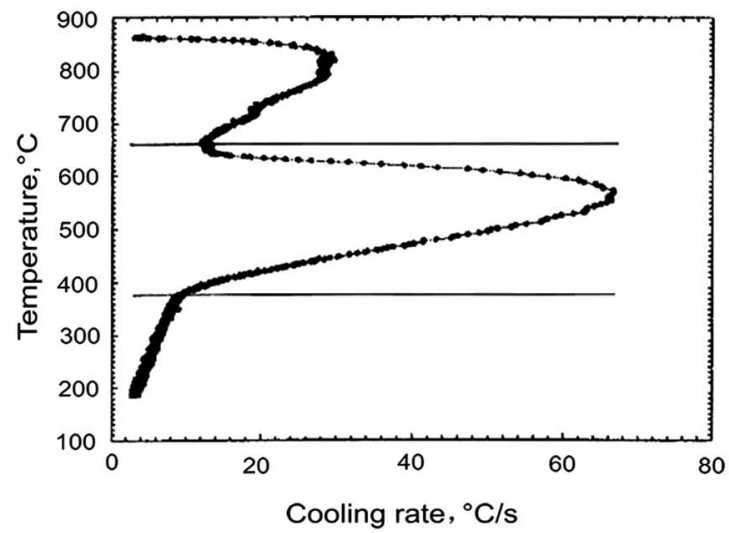

(a)

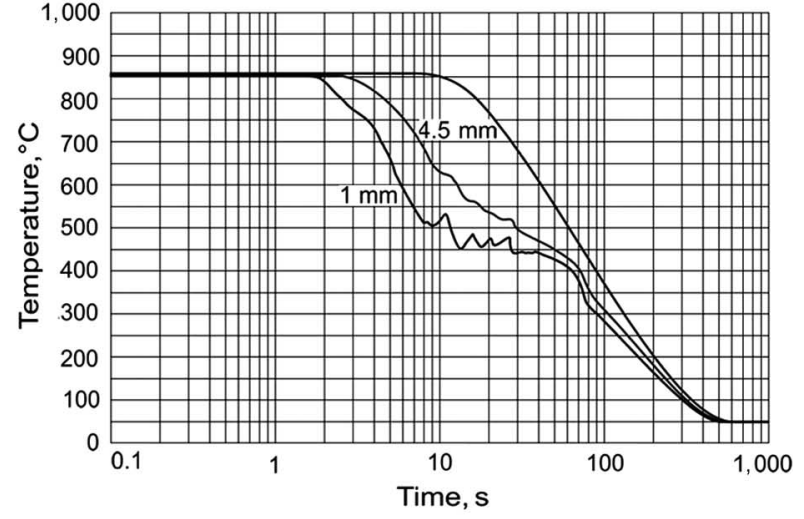

(b) 
behavior and transformation behavior of an actual workpiece when quenching in this kind of medium requires a more comprehensive representation.

Fig. 7 shows that in an actual quenching process, cooling exhibits dynamic characteristics. In the cited study [32], the authors measured the dynamic cooling behavior of conventional carbon steel bars that were $5 \sim 8 \mathrm{~mm}$ in diameter and were equipped with a center thermocouple into tap water, $5 \%$ brine, and $0.5 \%$ aqueous solution of carboxymethy cellulose (CMC), a water-soluble polymer, to evaluate the cooling rate variation with respect to time.

Fig. 7a shows the instantaneous cooling curve that was obtained when quenching a 6.5-mm probe into a vaporizable liquid medium, which, in this case, was tap water [33]. The waveform of the signal potential fluctuation shows the characteristic time domain during the quenching process, which indicates the changes that occur in the cooling process with respect to time. According to the principle of dynamic measurement, the dynamic cooling curve should reveal the rate of change of the cooling rates. A complete oscillation waveform for a dynamic curve is equivalent to the process of an increasing and decreasing cooling rate-time diagram where each $+/-$ variation from zero means the direction of the cooling rate curve is reversed. Several oscillations over zero indicates the strong dynamic changes in cooling rate that are measured by the probe in the high-temperature range. On the dynamic curve, the vapor blanket stage and the bubble boiling stage cannot be separated completely, indicating the instability of the cooling process in the high-temperature stage. As the oscillations tend to flatten, cooling enters the convection phase, and the dynamic characteristics decrease, but the cooling rate is still changing. Therefore, the dynamic cooling rate test can detect the instantaneous change of the cooling rate in a quench-cooling process to some extent. Because the probe diameter is relatively small and can be cooled entirely during the quenching process while being monitored, the measured results can explain, to some extent, the cause of the fluctuation of the surface cooling curve measured by Liščić in Fig. 6b [32].

To clarify the problem, a comparison between cooling time-temperature curves, cooling rate curves and their dynamic characteristics for different cooling modes are summarized in Table 1.

\section{FIG. 7}

A typical dynamic cooling curve measured with a $\Phi 6.5-\mathrm{mm}$ rod in (a) tap water, (b) $5 \%$ brine, and (c) $0.5 \%$ CMC aqueous solution.

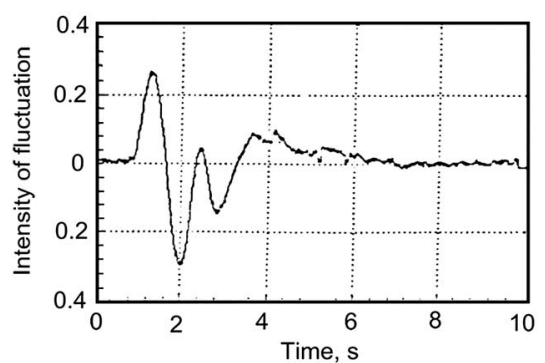

(a)

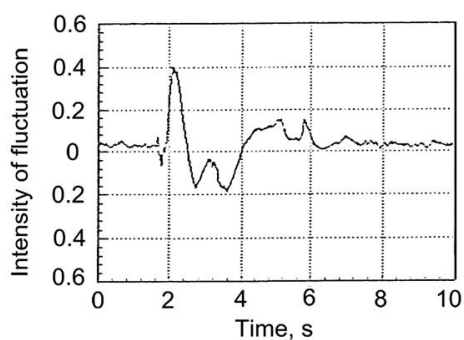

(b)

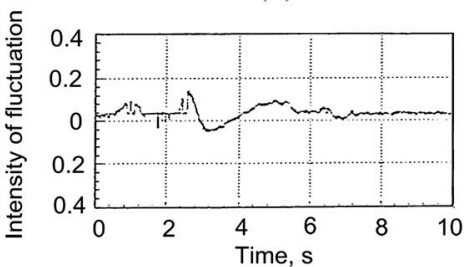

(c) 
TABLE 1

The function characteristics of different cooling models.

\begin{tabular}{lccc}
\hline Cooling Mode & Cooling Curve & Cooling Rate Curve & Dynamic Feature \\
\hline TTT & $T=f(\tau)=\Delta t$ & $v=T^{\prime}=0$ & $/$ \\
CCT & $T=f(\tau)=-c \tau$ & $v=T^{\prime}=c$ & $v^{\prime}=0$ \\
ACT & $T=f(\tau)=f(\mathrm{x}, \mathrm{y}, \mathrm{z}, \tau)$ & $v=f^{\prime}(\mathrm{x}, \mathrm{y}, \mathrm{z}, \tau)$ & $v^{\prime}=f^{\prime \prime}(\mathrm{x}, \mathrm{y}, \mathrm{z}, \tau)$ \\
\hline
\end{tabular}

Note: $\quad$ TTT $=$ Time-Temperature-Transformation curve; CCT $=$ Continuous Cooling Transformation curve; $\mathrm{ACT}=$ Actual Cooling Transformation curve; $T=$ the relationship between temperature and time; $\tau=$ time, $\mathrm{s}$; $\Delta t=$ temperature differential between austentizing temperature and transformation temperature; $v=$ cooling rate, ${ }^{\circ} \mathrm{C} / \mathrm{s} ; T^{\prime}=$ the derivative of temperature-time function, ${ }^{\circ} \mathrm{C} / \mathrm{s} ; c=$ a certain constant cooling rate, ${ }^{\circ} \mathrm{C} ; \mathrm{x}, \mathrm{y}, \mathrm{z}=\mathrm{a}$ certain position coordinate in a workpiece being quenched.

Table 1 shows that the cooling mechanism of the three cooling models is different just as the results of the cooling process are completely different. Because of a mass (volume) effect, wall thickness difference, medium characteristics for real workpieces during an immersion-quenching process, including all three cooling stages, the workpieces will experience complex cooling rate changes, and the resulting stress that changes the quench-related distortion would be expected. Therefore, in order to solve the distortion problem, which may occur during immersion quenching, with three-stage cooling, it is necessary to simplify the complex heat transfer phenomenon, which is beneficial for both cooling and distortion control.

During the actual steel-quenching operation, an important consideration is to guarantee the shape and size of the parts within a specified range, in addition to obtaining a martensitic microstructure. Therefore, it is necessary to associate the cooling mode or cooling medium characteristics with the effect of thermal stress and transformation stress on distortion during the quenching process that relates to "cooling uniformity."

Therefore, when distortion must be considered in the quenching process, it must be considered from two aspects, i.e., material nature and cooling coordination. The former is used to improve the stability of the austenite by selecting the alloy system so that the steels with higher hardenability are applied to parts that are consistent with the service requirements, and the latter is to select a cooling medium with as low a quench severity as possible to reduce the complex stresses during the phase transformation. If there are no suitable materials to ensure the required size precision for the parts to be quenched, then the only recourse is the selection of a cooling medium of optimal severity. In this case, the hardness must be limited, rather than the highest hardness, which may be obtained when using quenching media with relatively high quench severity. According to the three-stage cooling model and the main-auxiliary interface cooling model of vaporizable quenching medium, and in search for a moderate and effective quench-cooling medium or method, it is necessary to develop and optimize each cooling stage of the three-stage heat transfer model. Therefore, it is not sufficient to simply immerse workpieces into water when quenching in the heat treat shop, but quenching methods or devices must be appropriately designed to achieve the desired hardness with minimal distortion.

\section{AIR COOLING/FAN (BLOWER)-COOLING}

This process is primarily used for quenching high-alloy distortion-free steel and highpressure gas quenching of alloy steel in vacuum furnace systems. Previously, it was necessary to use the low-vapor pressure vacuum quenching oil for quenching in the low-pressure carburizing process, but now it is possible to utilize high-pressure nitrogen or helium injection methods so that carburized workpieces can be quenched fast and 
FIG. 8

Cooling curves of different kinds of high-pressure gas quenching.

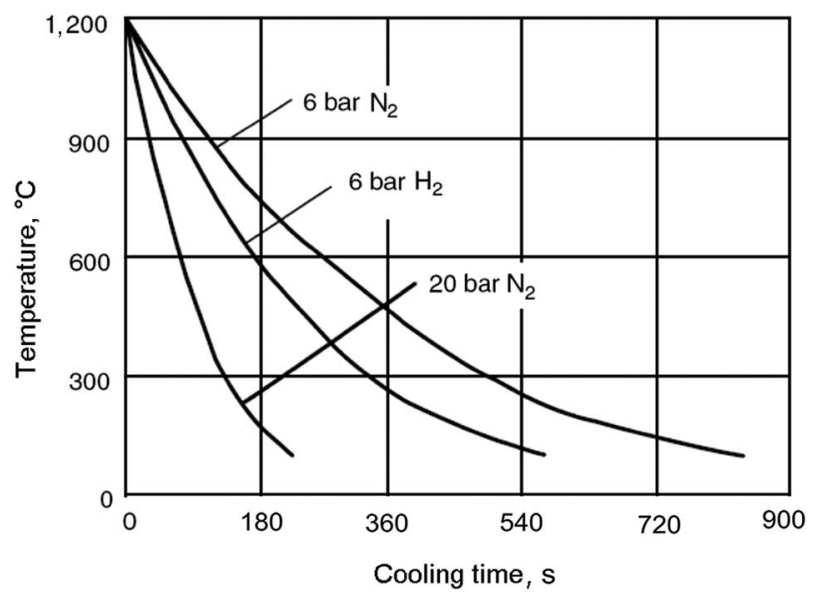

uniformly to inhibit deformation or cracking. This process provides a good example of the use of a single gas phase on the free interface without the constraints of an auxiliary interface and the surrounding bulk liquid. Fig. 8 shows the cooling curves for different cooling curves obtained for high-pressure gas quenching, which have obvious characteristics of a single convection cooling stage [34].

\section{SALT BATH QUENCHING BASED ON CONVECTION COOLING}

When molten salt baths are used as a cooling medium, they do not exhibit a phase change during the quenching process and therefore are suitable for some specialized heat treatment operations. For "clean" heat treatment production, fundamental changes for on-site management of these facilities are necessary. Nevertheless, molten salt quenching provides a good example of the use of the principle of conduction and convection directly on the free main interface during the cooling process.

Other methods, including molten metal baths, are also used for single-phase cooling. Fig. 9 illustrates the cooling curve of a molten lead patenting bath with a single convection cooling phase, where the cooling speed is continuous, and a low quench severity transition with decreasing temperature.

\section{FLUIDIZED BED}

Fluidized bed cooling is a man-made two-phase flow because it can obtain a pseudo liquefaction effect, which can also be considered as a type of single-phase cooling medium where its cooling capacity, adaptability, and applicability possess considerable development potential. Fig. 10 shows an application of a fluidized bed. Fig. 10a is the patenting treatment of high-strength steel wire using fluidized bed cooling whose cooling curve, Fig. 10b, exhibits single-phase cooling characteristics $[35,36]$.

\section{Evolution from Cooling Model to Cooling Engineering Characterized by Cooling Uniformity}

According to the cooling uniformity requirements on quench-hardening of steel, and on the basis of the understanding of the cooling model and cooling characteristics of 
FIG. 9

Cooling curve and cooling

characteristic curve of stee

wire during patenting in

lead bath.

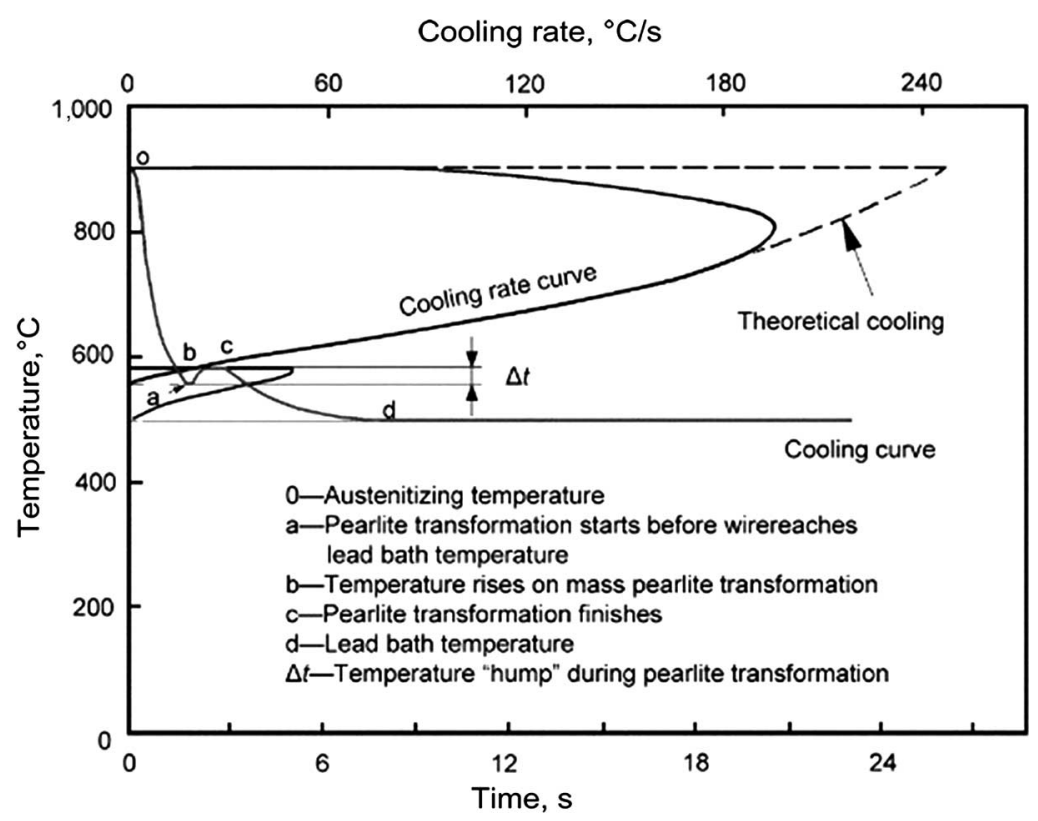

FIG. 10 Schematic of a wire patenting process using a fluidized bed: (a) wire patenting line and (b) cooling characteristic of fluidized bed.

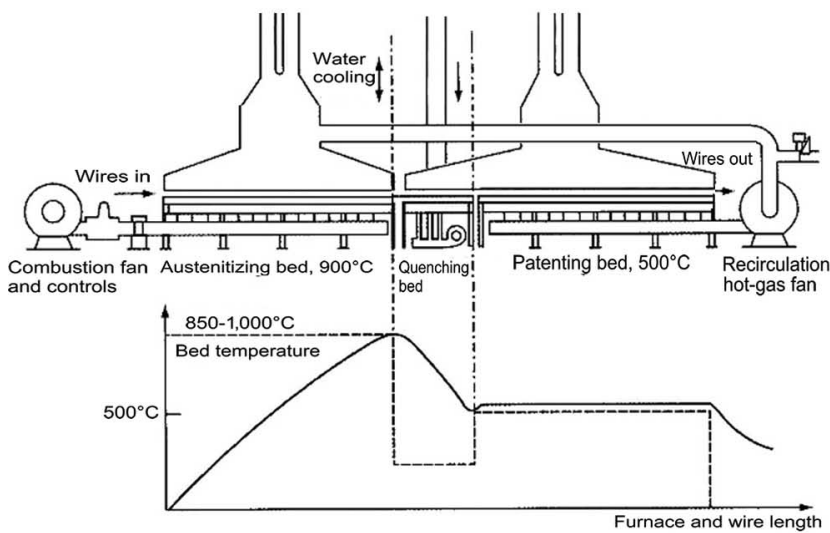

(a)

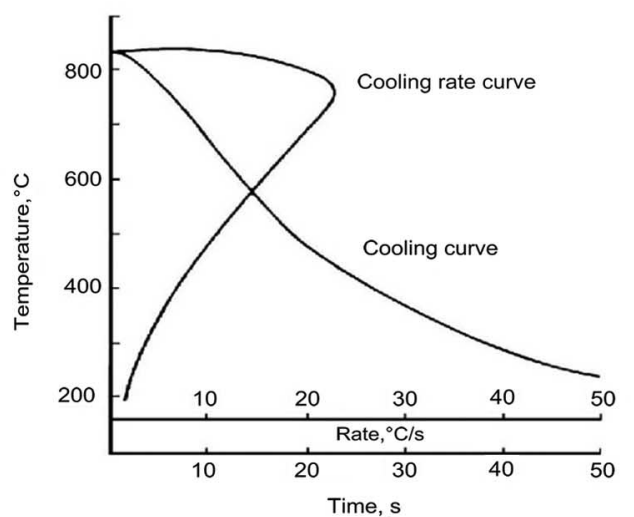

(b)

vaporizing quenchants, engineering application research is developing and is mainly aimed at the controllability of cooling. Therefore, the new cooling engineering model should focus on the consistency of microstructure transformation and distortion control on quenching instead of simply looking for the so-called best quenching media that conforms to the three-stage cooling model.

From the comparison of cooling curves and characteristic curves of TTT, CCT, and Actual Cooling Transformation (ACT) summarized in Table 1, it can be known that the cooling mode determines the transformation behavior of steel. However, in the actual workpiece quenching process, the specific components of quenching transformation 
(ACT) law upon cooling is very different from those of the construction conditions of TTT or CCT diagrams. In the future, it will be important to deepen the general understanding of the cooling transformation law of specific components according to the combination of available cooling media and technology in order to solve the problems of cooling uniformity and to minimize or eliminate the cracking, distortion, unreasonable residual stress, and uneven hardness of the parts during the quench-cooling process; that is, the ultimate uniformity of the quenching process must be pursued.

One of the most influential factors of quenching uniformity is the design of a quenching cooling system rather than simple quenching medium selection. George Totten, one of the authors of this article who used to be the chairman of the quenching cooling and distortion control board of the IFHTSE, believes quenching is still a "black hole" in heat treatment, in which there is still too much to be known [37]. He emphasized that agitation of quenchant is one of the most important research fields in the design of quenching cooling systems. Therefore, the design of a good mixing system for the cooling process of specific components may be one of the most promising ways to achieve homogeneous quenching effects. He also pointed out that research on fluid type, fluid concentration, agitating rate, quenching chute design, and quenching intensity of agitator and other variables would be continued, which implies that the quenching device design has the same importance as the quenching medium development.

\section{THEORY EXPLORATION AND TESTING TECHNOLOGY}

The standard probes currently in use can measure and compare the static cooling characteristics of a cooling medium. However, the test results are not necessarily related to the actual quenching effect of the steel workpieces under production quenching conditions in the workshop. Actually, there is no "typical" cooling curve in a real quenching process. An objective of the quenching process is to achieve stability of the geometry shape and size of the components to be quenched and the heat flux at the cooling interface, which are both dependent on the heat transfer mode and control throughout the quenching process. Development of a new quench-cooling theory and model, and making use of quenching medium characteristics, is important for the advanced heat treatment technologies. Therefore, the research on basic theory and model of quench-cooling engineering is related to the following:

- The transformation behavior and latent heat of austenitic phase transformation of different steel grades under actual cooling conditions;

- The influence of the cooling characteristics of the quenching medium on the hardening quality of the workpiece to be quenched;

- The effects of concentration, temperature, and agitation of aqueous polymer quenchants on quenching intensity;

- The development of new cooling media;

- The internal stress measurement and control of quenching intensity of a workpiece during quenching; and

- Computer simulation of quenching processes and establishment of appropriate mathematical models.

The development and optimization of these factors are vitally important for perfecting quenching processes, minimizing distortion and cracking of workpieces, and improving the overall quality of workpieces after quenching. In order to understand the synergistic behaviors of quenchant on an actual component during an actual industrial 


\section{FIG. 11}

(a) Sketch of the Liščić/Petrofer probe with the handle, and

(b) photo of the Liščić/Petrofer probe.

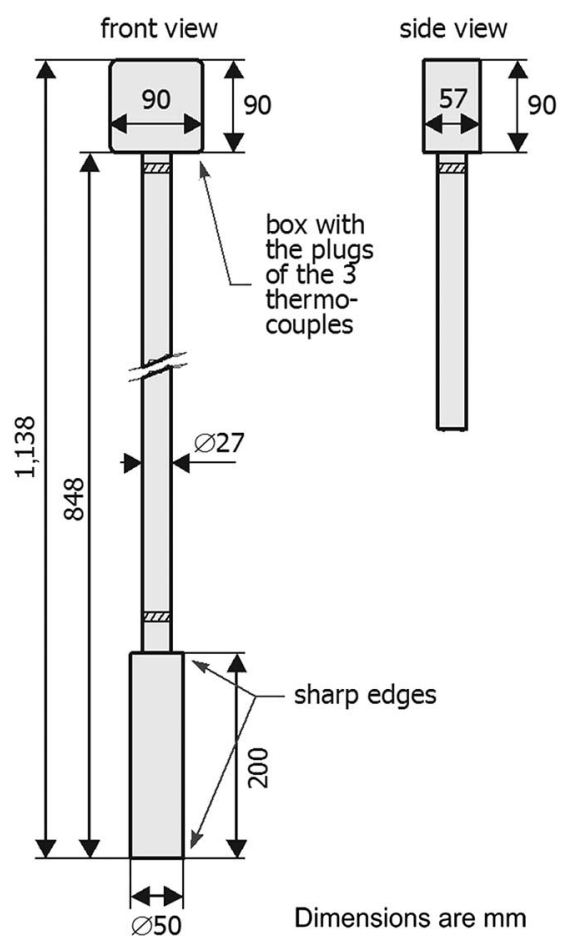

(a)

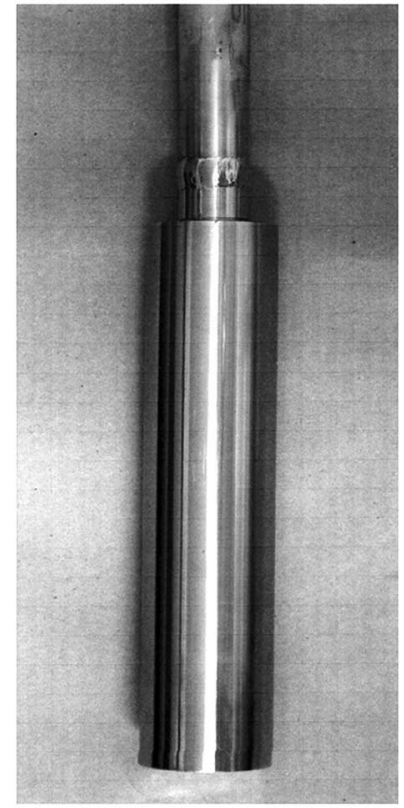

(b)

workshop quenching process, a methodology of quenchant characterization has been proposed by Liščić and Kobasko and Liščić, respectively, in Refs. [38,39]. Fig. 11 illustrates the probe developed by Liščić for quenchant characterization, which is called the Liščićl Petrofer probe.

The Liščić/Petrofer probe is a solid cylinder with a $50-\mathrm{mm}$ diameter and $200-\mathrm{mm}$ length. Fig. 11a shows the schematic of the probe with the handle, and Fig. 11b is a photo of the probe itself. The ratio between length and diameter, $\mathrm{L} / \mathrm{D}=4: 1$, ensures that the heat dissipation through both ends of the probe is negligible so that in the cross section at the half length of the probe where the thermocouples (TCs) are positioned, only radial heat flow exists. This is a prerequisite for 1-D heat transfer calculations. Because of its cylindrical shape, the Liščić/Petrofer probe is applicable for heat transfer calculations of axially symmetric workpieces with diameters between 20 and $100 \mathrm{~mm}$ [38]. The Liščić/Petrofer probe is constructed of INCONEL 600, a nickel-chromium austenitic alloy, which, during heating and cooling, does not undergo structural transformation and is oxidation resistant [39]. The probe is instrumented with three sheathed and grounded TCs: one is placed $1 \mathrm{~mm}$ below the surface, the second one is placed $4.5 \mathrm{~mm}$ below the surface, and the third one at the center of the cross section at the half length of the probe. The curves for heat flux density and heat transfer coefficient at different temperatures or times on the surface can be calculated. Under the heat flux curve, the area within a certain time interval represents the heat discharged from the probe quenching, which can be used as a criterion for evaluating the quenching intensity of real workpieces. 


\section{RELATIONSHIP BETWEEN COOLING PERFORMANCE TEST RESULTS AND HEAT TREATMENT EFFECT}

The cooling characteristics of the cooling medium under laboratory testing conditions can be evaluated using a nickel alloy probe (Inconel 600) or a silver probe such as that described in the Japanese standard K 2243, Heat Treating Fluids [40,41] or ASTM D7646, Standard Test Method for Determination of Cooling Characteristics of Aqueous Polymer Quenchants for Aluminum Alloys by Cooling Curve Analysis [42]. In this way, the cooling performance test results of different quenching agents under standard (nonindustrial) conditions can be compared, but the correlation between the cooling rate of industrial quenching equipment with different degrees of agitation has not been established. Therefore, in the international heat treatment industry, the correlation of the cooling characteristics measured by the laboratory standard probe method with the actual quenching effect under real quenching conditions is yet unresolved, although various attempts have been made to resolve it.

Fig. 12 illustrates the results of a study by Segerberg to try to link the upper and lower characteristic points on a cooling characteristic curve obtained by the ISO 9950 testing procedure with the average cooling rate at the nose of the CCT diagram to express the quenching or cooling capacity of quenching oils. Eq 1 is the regression equation obtained after testing a wide variety of petroleum quenching oils [43].

$$
H P=91.5+1.34 T v p+10.88 C R-3.85 T c p
$$

where:

$H P=$ quenching hardening ability, a dimensionless parameter reflective of the as-quenched hardness;

$T v p=$ upper characteristic point, ${ }^{\circ} \mathrm{C}$;

$C R=$ the average cooling rate between $500^{\circ} \mathrm{C} \sim 600^{\circ} \mathrm{C},{ }^{\circ} \mathrm{C} / \mathrm{s}$; and

\section{FIG. 12}

Illustration of the characteristic parameters obtained from a standard cooling timetemperature curve.

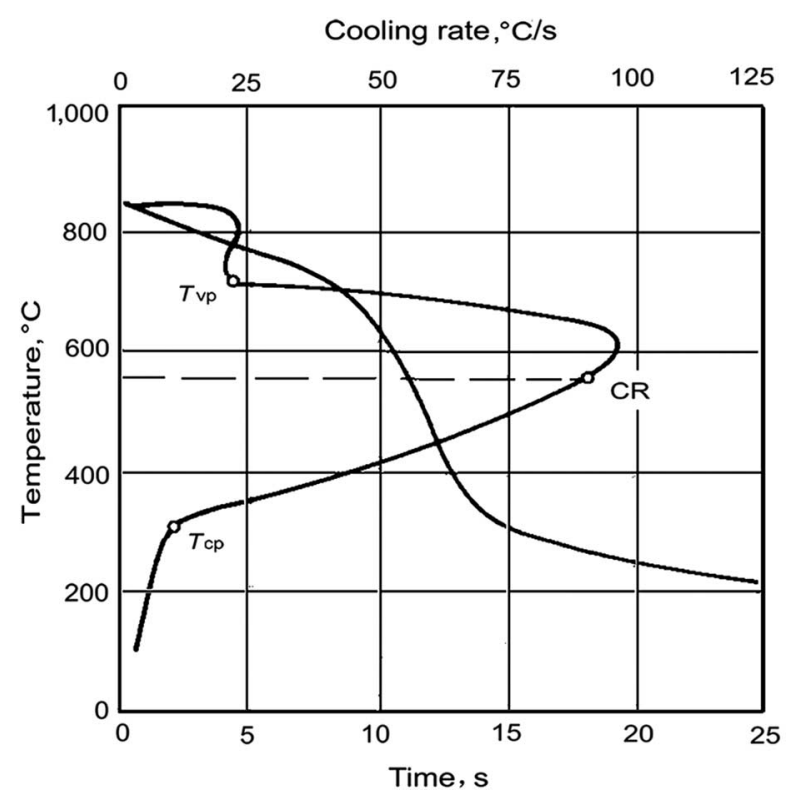


$T c p=$ the next characteristic transition temperature for the transition of nucleate boiling to convective cooling, ${ }^{\circ} \mathrm{C}$.

The objective of Segerberg's work was to establish a connection between the data obtained from the cooling curve measurement and the hardening effect of the steel components after quenching. Segerberg used a carbon steel for his hardness correlation work. Subsequent to this work, Zeng [44] and Chen and Zhou [45] performed additional studies on the Segerberg correlation. Chen and Zhou, for example, showed that the Segerberg correlation provided better agreement with test results than the transition temperature from the vapor blanket cooling stage (full-film boiling) to the nucleate boiling stage and the maximum cooling rate. The hardening power equation also provided better agreement with respect to hardness for carbon steel than for alloy steel. As a result of their work, a set of revised hardening power $\left(H P_{C}\right)$ equations for carbon and alloy steels was developed. Eqs 2-4 were developed for plain carbon steels:

$$
\begin{gathered}
H P_{C}=10\left[V_{m}-0.25\left(T_{m}-550\right)\right]+10 V_{300}, \text { where } T_{m}>550^{\circ} \mathrm{C} \\
H P_{C}=10\left(V_{m}-0\right)+10 V_{300}, \text { where } 500^{\circ} \mathrm{C} \leq T_{m} \leq 550^{\circ} \mathrm{C} \\
H P_{C}=10\left[V_{m}-0.25\left(500-T_{m}\right)\right]+10 V_{300} \text {, where } T_{m}<500^{\circ} \mathrm{C}
\end{gathered}
$$

For these equations,

$H P_{C}=$ Corrected Segerberg $H P$ value;

$V_{m}=$ maximum cooling rate, ${ }^{\circ} \mathrm{C} / \mathrm{s}$

$T_{m}=$ temperature at the maximum cooling rate, ${ }^{\circ} \mathrm{C}$; and

$V_{300}=$ Cooling rate at $300^{\circ} \mathrm{C}$.

A second set of regression equations was developed for alloy steels:

$$
\begin{gathered}
H P_{C}=10\left[V_{m}-0.25\left(T_{m}-500\right)\right]+10 V_{300}, \text { where } T_{m}>500^{\circ} \mathrm{C} \\
H P_{C}=10\left(V_{m}-0\right)+10 V_{300}, \text { where } 450^{\circ} \mathrm{C} \leq T_{m} \leq 500^{\circ} \mathrm{C} \\
H P_{C}=10\left[V_{m}-0.25\left(450-T_{m}\right)\right]+10 V_{300}, \text { where } T_{m}<450^{\circ} \mathrm{C}
\end{gathered}
$$

These equations show that there is an alloy-dependent optimal quenching temperature range. These revised equations were reported to be superior to Segerberg's original Hardening Power Equation for unagitated petroleum quenchants (Eq 1).

\section{DEVELOPMENT OF NEW COOLING TECHNOLOGY}

At the present time, current research related to quench-cooling technology involves the study of computational fluid dynamics (CFD) and digital simulation technology [46]. Fig. 13 illustrates the results of a CFD study of the simultaneous velocity and temperature distribution in a quenching tank with heating and stirring systems. The objective of this study was to determine the source of nonuniform quenching results being obtained. A 3-D flow field distribution of quenching medium in the quenching tank was calculated and the "effective quenching zone" [47] in the quenching tank was determined by analyzing the flow characteristics of the cooling medium in the quenching tank and the influence of the agitation device (a single top-entering impeller) on the flow field distribution. Ideally, in the effective quenching zone, a uniform area of flow cooling would ensure that the workpiece obtained the required microstructure and properties that 
FIG. 13 CFD diagram in quenching chute with heating and agitation system.

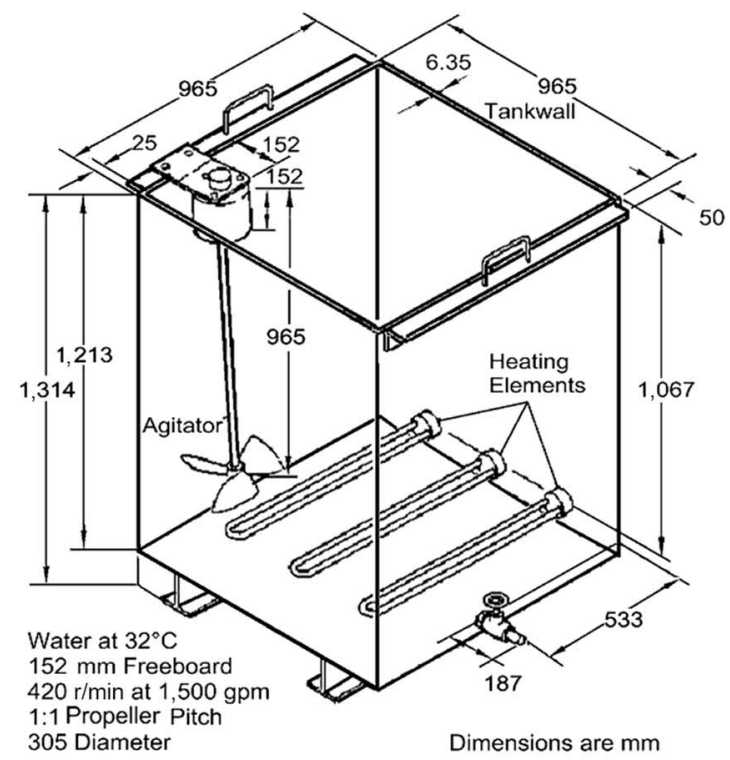

(a)

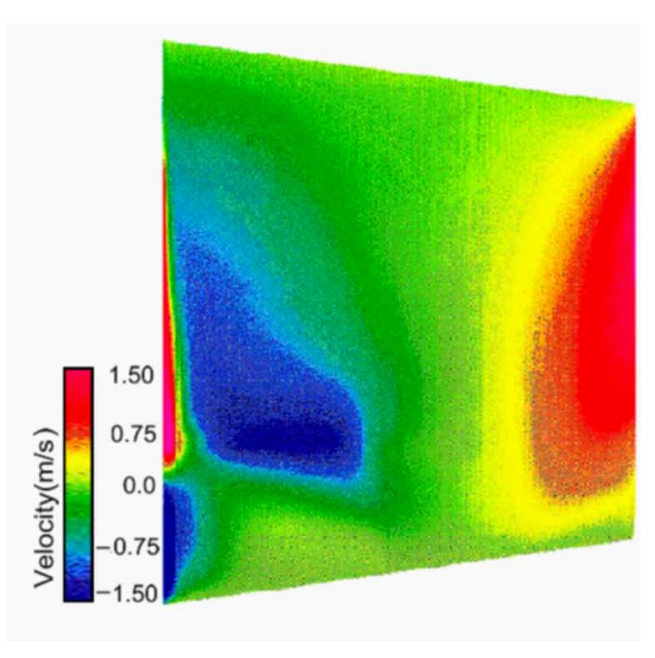

(b)

would result in improved quenching quality. The simulation of the cooling medium flow field in the 3-D quenching tank provided the overall distribution of velocity vector and flow field at any position in the tank and also provided a useful reference for determining the effective quenching area of the quenching tank in the quenching operation. The impact of this study was to not only show the nonuniformity of flow present in the tank, which led to the wide distribution of as-quenched results, but also to show the impact if the main-auxiliary interface model considered making improvements in the accuracy of the simulation.

While the previous example is a relatively early example of the use of CFD analysis to troubleshoot a poorly performing small quenching system, this methodology is now becoming increasingly common for use in quench system optimization and design. CFD analysis was used to develop optimal racking configuration for a system used for the quenching of carburized AISI 8620 steel pinions by identifying several locations where the flow could be improved and to produce improved hardness uniformity [48]. Chen utilized an ultrasonic Doppler velocimeter to measure the flow rate of quenchant in a large tank with draft-tube agitators. CFD analyses were then performed to simulate the optimal flow distribution by the baffle positioning in the quench zone [49]. In a different study, Bineli et al. developed two models of agitation for an industrial rectangular quench tank; one system had a submerged agitation system located on the bottom of the tank, and the other had the addition of a lateral flow arrangement [50]. This simulation involved determining the cooling profile and heat transfer coefficient of the parts being quenched to determine the agitation system that will produce the greatest flow uniformity which, in turn, would produce optimal mechanical properties of the parts. Banka et al. used CFD analysis to study water flow fields and surface heat transfer rates for a gear that was intensively quenched. This work first involved determination of the quenchant temperature 
and heat transfer coefficients along the gear surfaces for use in predicting the fixture system placement to produce optimal uniformity in the metallurgical, stress, and distortion responses of the gear [51].

CFD simulation was used to develop an analytical equation for cooling time evaluation for the process of quenching semiaxles and cylindrical forgings in an agitated water tank. The advantage of CFD technology is that it does not require knowledge of the heat transfer coefficients at the surface of steel parts. The results of this work showed that a generalized correlation can be used to calculate the cooling time for both simple and complicated configurations [52]. Nonuniform agitation will produce unacceptable nonuniform as-quenched properties. Heat transfer coefficient variation produced in a quench tank has been examined by quench process simulation using CFD analysis. In this study, the authors proposed process variations that would yield optimal quench uniformity [53].

Based on fluid dynamics and stress control research, i.e., the results of quenching technology, intensive quenching methods are being developed by Kobasko, Aronov, and Powell [54]. Fig. 14 shows an intensive quenching device used for shaft components. In this case, intensive quenching methodology utilized a high-pressure spray-quenching medium where the aqueous quenchant is strongly sprayed directly on the workpiece surface by controlling the pressure, flow, and proportion of the spray-quenching medium. According to the physical metallurgical transformation principle and stress state of the workpiece and adjustment of the cooling process time, the method can create uniform cooling and produce a greater hardened depth, more uniform surface hardness, and minimal distortion for high-quality workpieces by obtaining maximum consistency between the material properties and quenching cooling effect, all while minimizing distortion. One quench process design method by which this is accomplished is by eliminating vapor blanket formation (film boiling) and minimizing nucleate boiling and facilitation of convective cooling - a single-phased process.

Generally, the intensive quenching processes are classified into three major categories [55,56]:

FIG. 14

A typical intensive quenching

system for shaft-like

components.

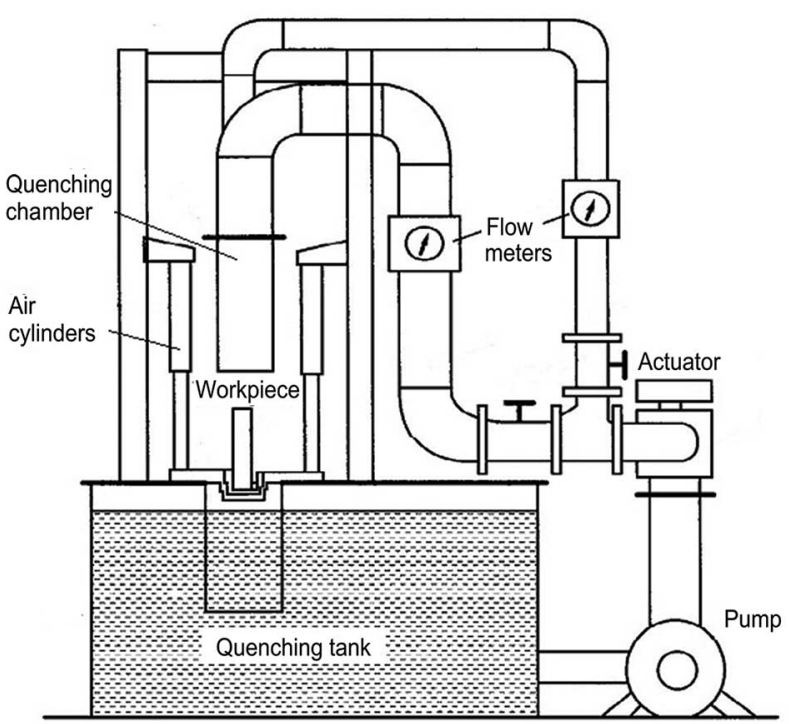


(1) IQ-1: Controllable three-stage cooling (program-controlled three-stage cooling);

(2) IQ-2: Controllable post-two stages of cooling (inhibiting vapor blanket boiling, only bubble boiling and convection stages);

(3) IQ-3: Controllable convection cooling (a single phase of forced convection).

Fig. 14 illustrates an intensive quenching device suitable for the quenching of shaft components.

According to Kobasko's IQ principles, computer modeling and simulation may be used for process design. The simulation program utilizes finite element methodology to analyze the change of temperature field and stress field during the quenching process and the time of obtaining maximum surface compressive stress is calculated. The calculation results include temperature field, microstructure field, stress field, distortion distribution, and quenching cooling process [57].

\section{QUENCHING DEVICE DESIGN (DIGITAL, CONTROLLABLE, AUTOMATIC, AND FLEXIBLE)}

Modern quench-cooling system designs not only involve cooling medium selections but also complete system designs. Special attention should be paid to the actual conditions of quenching components and their cooling requirements in order to achieve a controllable quenching effect. This effect includes the controllable cooling process, the maximum material properties, ultra-performance for service, and a full guarantee of component dimensional accuracy to meet the current and future demands for advanced manufacturing goals. Fig. 15 shows a quenching device for inner ring hardening of a precision heavy-duty bearing [58].

The bearing ring quenching device shown in Fig. 15 utilizes the Bernoulli principle of hydromechanics as shown in Fig. 16, which illustrates the flow diagram of fluid in a closed tube. Because the volume of the fluid in the closed tube cannot be compressed with the change of the section of the passage, the kinetic energy, potential energy, and pressure energy contained in the fluid will be definite but internally self-adjusting. However, because of the viscous nature of fluids, there is an unavoidable loss of energy that needs to be specially accounted for in the design for the closed passageway. According to the analysis of the quenching medium flow shown in Fig. 16, the design principle of the flow path of the cooling program is embodied by adjusting the flow of the cooling medium and the channel gap.

FIG. 15

Specially designed hardening apparatus for bearing of rail vehicle.

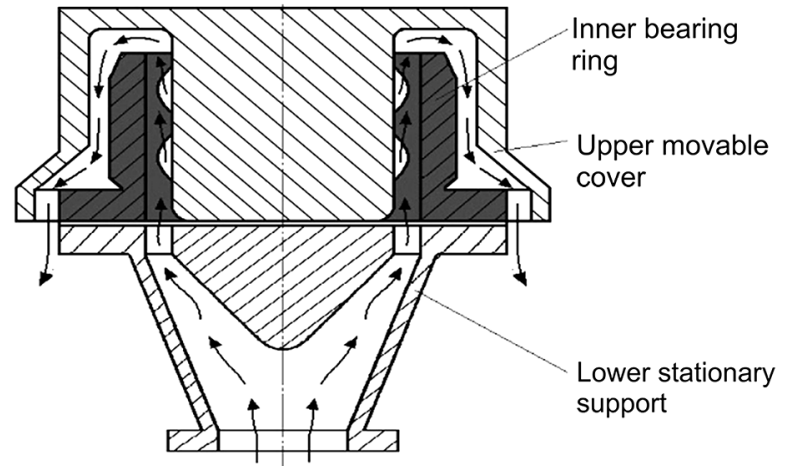

Quenchant flow 
FIG. 16

Fluid flow pattern in a closed tube with section area changes:

(a) Bernoulli flow diagram and

(b) simulation diagram.

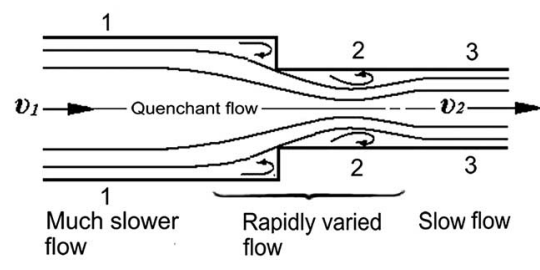

(a)

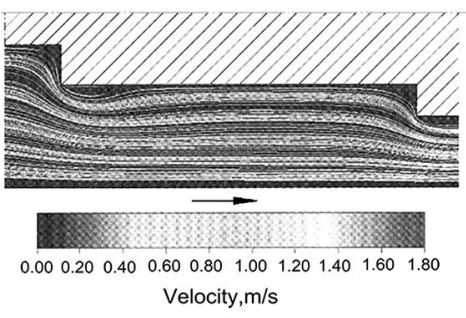

(b)

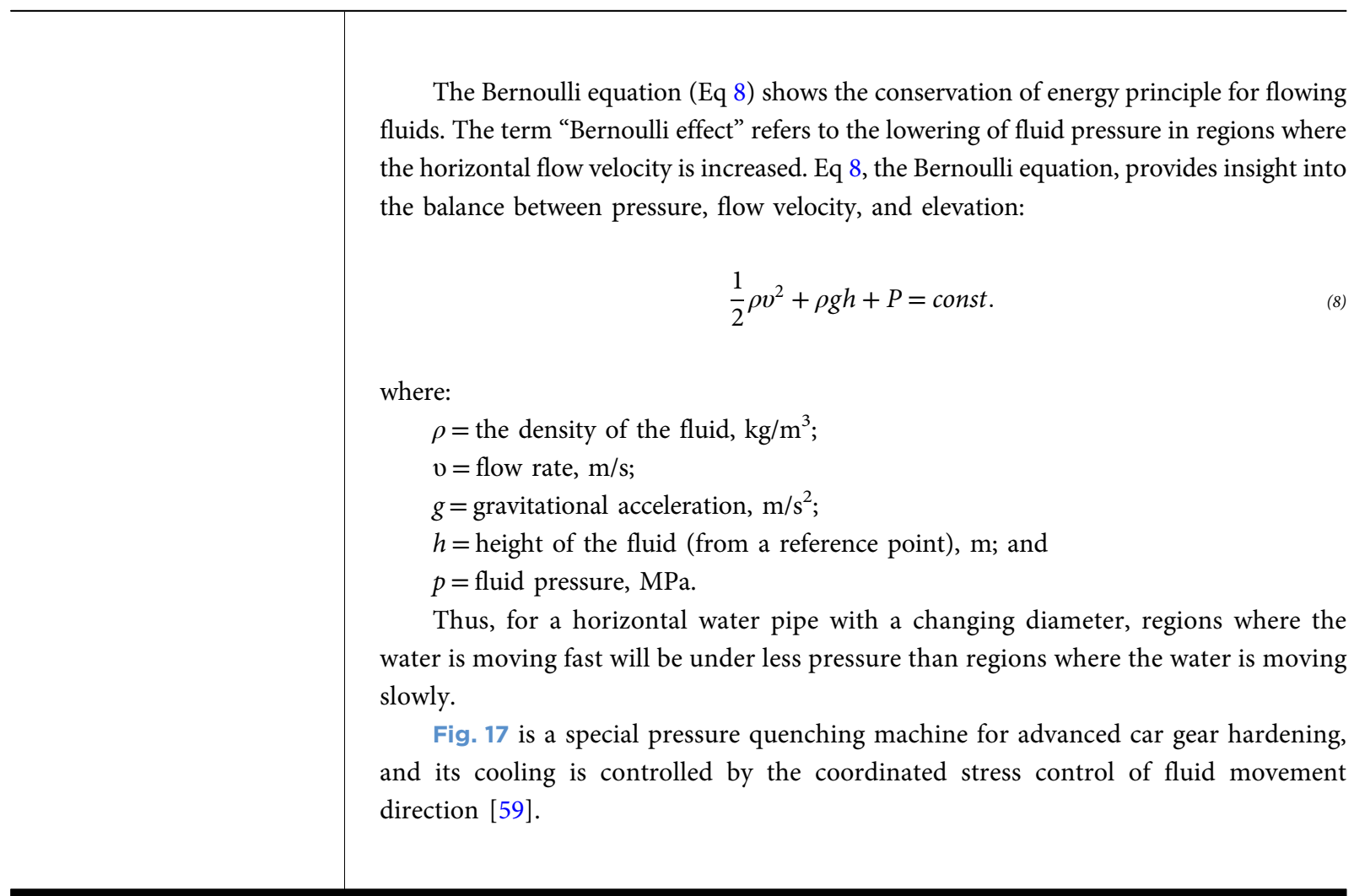

\section{FIG. 17}

Press quenching for bevel gears and the quenchant flow route:

1. Sealing shell, 2. Outer ring die,

3. Inner ring die, 4. Workpiece,

5. Universal lower die, and

6. Expanding mandrel.

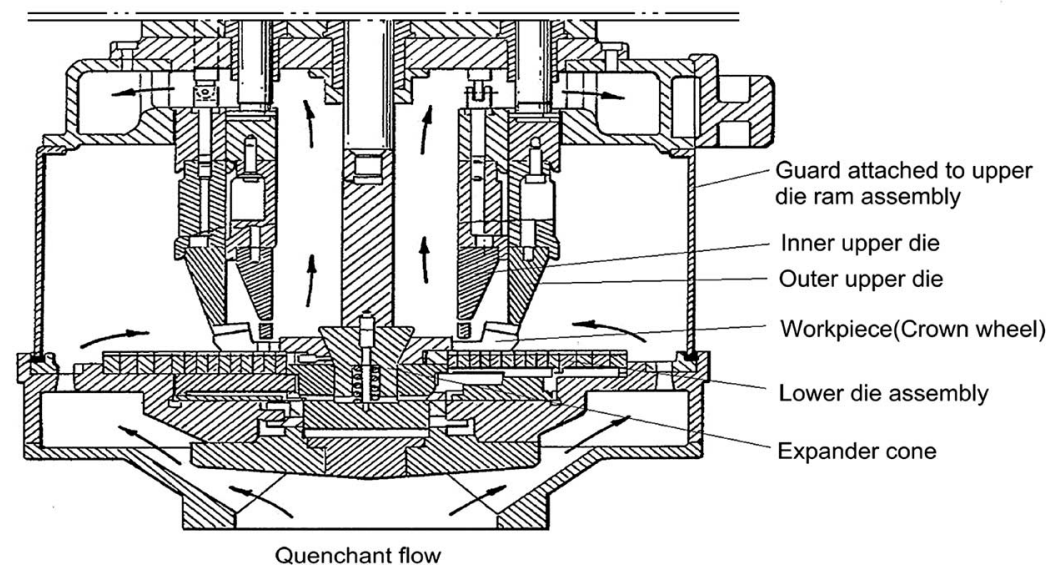




\section{CONTROLLABLE QUENCH-COOLING TECHNOLOGY}

Controllable quenching technology is a relatively new research area for quenchants and quench-cooling technology. It has been found that there are many ways to adjust the cooling process, and computer programmed automatic control technology is a critically important element in controllable cooling process development. Fig. 18 provides a schematic diagram of the cooling curve of surface, subsurface, and core of a large material section upon quenching and its simulation results [60]. The comparison between conventional quenching and controllable quenching is summarized in Table 2.

\section{ENGINEERING DESIGN OF QUENCH-COOLING FACILITY—INTEGRATION, INTELLECTUALIZATION, MATCHING, SERIALIZATION}

Integration means that some features are grouped together rather than one device with only a single function. Intelligence refers to making use of artificial intelligence and digital technology so that the system has a sensitive response to operation parameters changes, the effective judgment, adjustment, and implementation abilities during the quenching work, as well as the data access. Matching must meet the cooling requirements of the quenching process, in line with the production rhythm and productivity, and be able to be embedded in standard or nonstandard heat treatment production line. Serialization

\section{FIG. 18}

Cooling curves at different positions upon precooling and controlled-loop cooling during quenching.

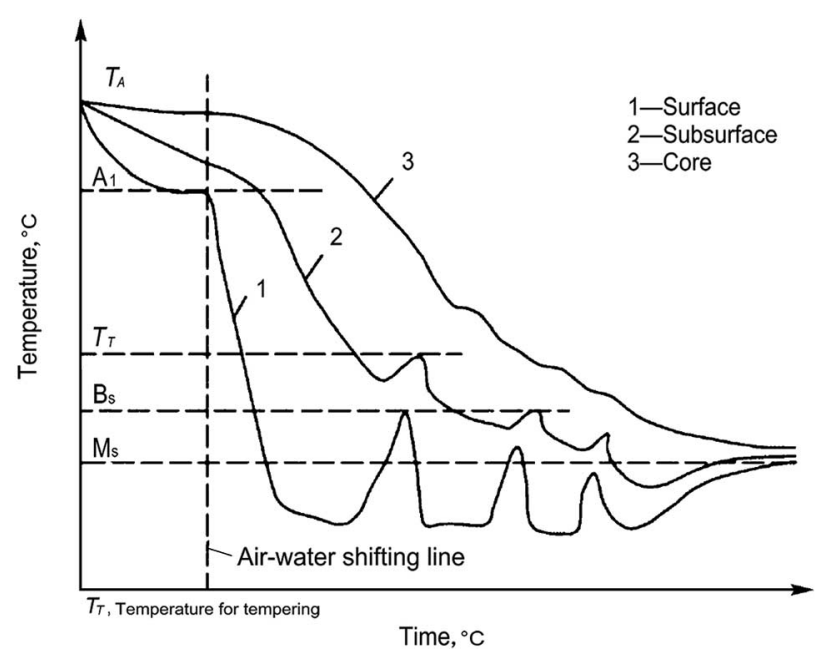

\section{TABLE 2}

Comparison between traditional quenching and controlled quenching.

\begin{tabular}{|c|c|c|}
\hline Item & Conventional & Controllable \\
\hline Medium & Water, oil, brine, salt bath & Air, water \\
\hline Cooling model & Three stages & Controlled fluid; controlled single-phase fluid; or controlled multiphase cooling \\
\hline Operation & $\begin{array}{l}\text { Hand-working, Quenching chute; } \\
\text { Interrupted, Mechanical moving; } \\
\text { Agitation; }\end{array}$ & $\begin{array}{l}\text { Computer simulation; Real-time measurement and control; Coolant selection; Multifactor } \\
\text { control-flow, pressure, cooling speed, flow mode; Specially designed quenching device }\end{array}$ \\
\hline Stress Control & Complex stress; Cracking & Residual compressive stress; Cracking-free \\
\hline Distortion Control & Difficult to control, Rely on correction & Using additional devices to control \\
\hline Application & Widely used & Developing, currently subject to a certain limit \\
\hline
\end{tabular}


FIG. 19

Schematic diagram of a type of multipurpose quenching system.

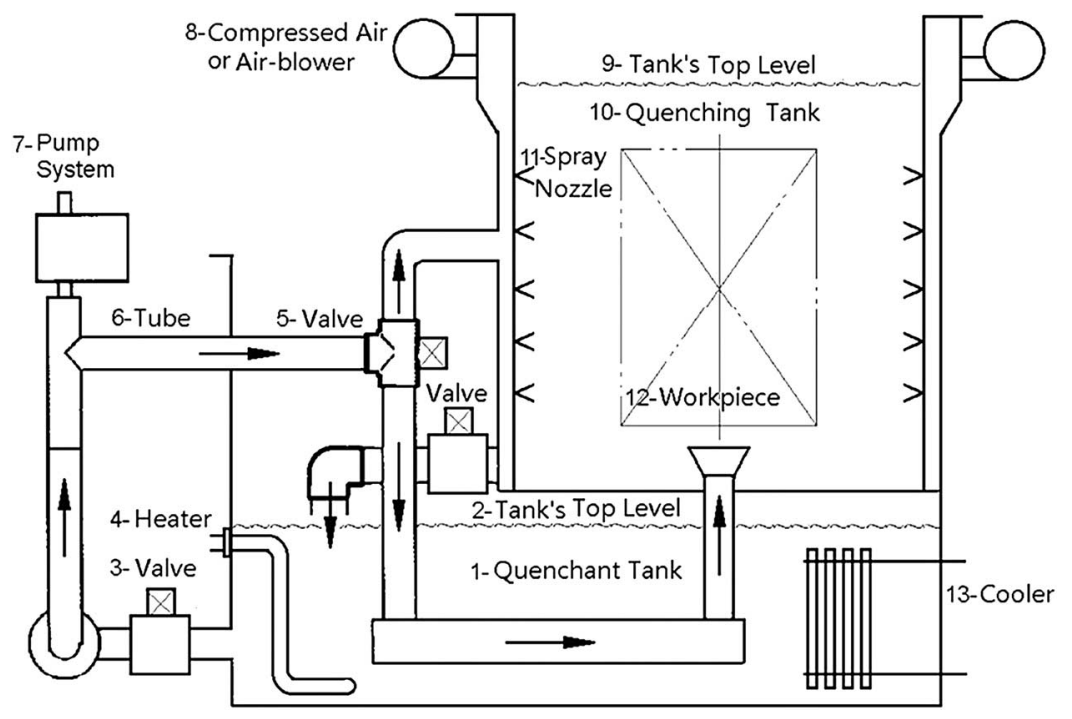

refers to the plan of the main types, parameters, dimensions, and basic structure of facilities based on the study of the development of quench-cooling technology and the prediction of its development trend.

Fig. 19 shows a multipurpose quench-cooling system with high performance, low energy consumption, and intelligent control [61] that is mainly used to quench disk or shaft components. On the basis of computer simulation of the cooling process, through programming control, it can realize accurately timed switching, complete combined quenching in real time, meet the cooling intensity requirement of each stage of the quenching process of specific parts, and control the degree of distortion within the permitted range. Basic functions of the system include immersion cooling, spray cooling, water-mist cooling, high-pressure air cooling, or a combination of some functions of these cooling methods.

The engineering method holds that the most critical and unique task of an engineer is to find, understand, and combine the limitations of actual conditions to achieve satisfactory results. In many cases of heat treatment, the equipment is designed to meet not only technical requirements but also other conditions, such as manufacturing conditions. In this way, modern heat treatment engineers should not only understand the basic principles and requirements of material heat treatment but also have extensive peripheral engineering experience in regards to material properties and sources required for equipment, parameter testing and process control, physical or technical limitations, feasibility of future improvements, and other factors, such as cost, availability, production effects, and applicability.

\section{Conclusions}

In metal (steel) heat treatment, it is relatively easy to meet the physical metallurgical requirements of the materials themselves, but the quench-cooling technology requirements for various non-equilibrium transformation products are the essence of modern 
heat treatment engineering. It is practical to solve quench-cooling problems and to design and develop systems required to meet the needs of advanced manufacturing industry by conducting system research from pure model analysis to "engineering" applications.

Three-stage theory on vaporizable quenchants during immersion quenching is a pure heat transfer model. TTT and CCT behaviors are physical metallurgical models. It is the actual model to combine the heat transfer model with quenching effect or metallurgical transformation model so that quench-cooling engineering can be achieved. Its core is controlled quench-cooling technology.

At present, the controlled quench-cooling technology is still in the primary stage. The corresponding basic theory has yet to be developed and improved, and numerical simulation of a large number of basic data and industrial large data need to be continuously supplemented. Digitally controlled quench-cooling technology is an important task in the research of quench-cooling technology.

There is no "best" quenching medium in heat treatment, only the best cooling technology. But a good cooling technology without modern equipment as a guarantee isn't industrially practical. Only by deepening the collective understanding of the physical metallurgy models and the "cooling engineering" concepts in metals heat treatment and establishing the appropriate facility support can the consistency of the quality of heat treatment products be achieved.

Finally, it is good to be aware of the damage that can be done to the practice by a too liberal and often unnecessary use of hypothetical models and concepts and an overexploitation of theoretical terminology [22].

\section{References}

[1] Hassan, A. A. and Hamed, M. S., "Toward the Achievement of Vision 2020 Goals of the Heat Treating Industry," JOM, Vol. 62, No. 9, 2010, pp. 55-59, https://doi.org/10. 1007/s11837-010-0138-Z

[2] Gaster, R. J., "ASM Heat Treating Society's 1999 Research \& Development Plan," 1999 Research Report, ASM Heat Treating Society, Materials Park, OH, 1999, pp. 1-20.

[3] Totten, G. E. and Albano, L. L. M., "IFHTSE Global 21: Heat Treatment and Surface Engineering in the 21 Century - Part 18: Quenching: Current Status and Future Developments," Int. Heat Treat. Surf. Eng., Vol. 6, No. 1, 2013, pp. 4-14, https:// doi.org/10.1179/1749514811Z.0000000001

[4] Fabian, R., "Heat Treating Community-To Achieve Vision 2020," 2001 Report, ASM Heat Treating Society, Materials Park, OH, 2001, pp. 1-8.

[5] Luo, X. M., Shao, H. H., Chen, K. M., Sun, J., and Totton, G. E., "Evolution from 'Cooling Model' to 'Cooling Engineering' (in Chinese)," Heat Treat., Vol. 29, No. 5, 2014, pp. 1-10.

[6] Luo, X. M. and Totten, G. E., "Wire Cooling Curve Analysis," ASM Handbook, Vol. 4B: Steel Heat Treating Technologies, ASM International, Materials Park, OH, 2014, pp. 310-313.

[7] Luo, X. M. and Totten, G. E., "Wire Patenting," ASM Handbook, Vol. 4A: Steel Heat Treating Fundamentals and Processes, ASM International, Materials Park, OH, 2013, pp. 257-261.

[8] Luo, X. M., Chen, K. M., and Totten, G. E., "Fast Primary Cooling: Alternative to Lead Baths for High-Carbon Steel Wire Patenting," Mater. Perform. Charact., Vol. 1, No. 1, 2012, pp. 1-13, https://doi.org/10.1520/MPC20120006

[9] Luo, X. M. and Totten, G. E., "Analysis and Prevention of Quenching Failures and Proper Selection of Quenching Media: An Overview," J. ASTM Int., Vol. 8, No. 4, 2011, pp. 1-29, https://doi.org/10.1520/JAI103397 
[10] Luo, X. M. and Li, J. K., "Effects of Cooling Rate Fluctuation on Cooling and Transformation Behavior of Steel upon Direct Quenching," J. ASTM Int., Vol. 6, No. 2, 2009, pp. 935-952, https://doi.org/10.1520/JAI101775

[11] Canale, L. C. F., Luo, X. M., and Totten, G. E., "Quenchant Characterization by Cooling Curve Analysis," J. ASTM Int., Vol. 6, No. 2, 2009, pp. 861-899, https:// doi.org/10.1520/JAI101981

[12] Luo, X. M. and Li, F., "Metallurgical Behaviors of High-Carbon Steel Wires in Lead Bath and CMC Aqueous Solutions by Cooling Curve Analysis," Int. J. Mater. Prod. Technol., Vol. 24, Nos. 1-4, 2005, pp. 142-154, https://doi.org/10.1504/IJMPT.2005. 007945

[13] Luo, X. M., "Cooling Behaviors of High-Carbon Steel Wires in Lead Bath and Alternatives for Patenting," presented at the Fourth International Conference on Quenching and Control of Distortion, Beijing, China, May 20-23, 2003, Chinese Heat Treatment Society, Beijing, China, pp. 247-252.

[14] Luo, X. M. and Zhu, S. X., "Merits of Small Probes in Research on Cooling Behavior of Steel upon Quenching," presented at the Proceedings of the 21st ASM Heat Treating Society Conference, Indiana Convention Center, Indianapolis, IN, Nov. 5-8, 2001, ASM International, Indianapolis, IN, pp. 225-229.

[15] Zhu, S. X. and Luo, X. M., "Experimental Research and Computerized Simulation on Carbon Steel's Quenching Process," presented at the 21st ASM Heat Treating Society Conference, Indiana Convention Center, Indianapolis, IN, Nov. 5-8, 2001, ASM International, Indianapolis, IN, pp. 220-224.

[16] Luo, X. M. and Li, J. K., "The Application of Dynamic Measurement to Exploration of Cooling Behavior of Steel upon Quenching," presented at the 20th Annual Conference on Heat Treating, St. Louis, MO, Oct. 9-12, 2000, ASM International, Indianapolis, IN, pp. 648-651.

[17] Luo, X. M., "Function of Polymer Additive (CMC/PVA) in Spray Quenching of Steel," presented at the International Symposium on Accelerated Cooling/Direct Quenching of Steels, Indianapolis, IN, Sep. 14-18, 1997, ASM International, Indianapolis, IN, pp. 247-251.

[18] Luo, X. M., "Influence of Probe and Its Cooling Behavior on Assessing Cooling Characteristics of Quenching," presented at the Second International Conference on Quenching and Control of Distortion, Cleveland, OH, Nov. 4-7, 1996, ASM International, Indianapolis, IN, pp. 503-508.

[19] Luo, X. and Liu, H., "Relations between Probe Design and Interpretation of Results for Quenching Media Test," J. Jiangsu Univ. Sci. Technol., Vol. 17, No. 5, 1996, pp. $50-55$.

[20] Liu, H. N. and Luo, X. M., "A New Process and Equipment for Direct Quenching of Gear Blank after Forging," presented at the International Heat Treating Conference: Equipment and Process, Schaumburg, IL, April 18-20, 1994, ASM International, Indianapolis, IN, pp. 463-468.

[21] Luo, X. M. and Liu, H. N., "Study on the Cooling Characteristics for Steel in Spray Quenching," presented at the International Heat Treating Conference: Equipment and Process, Schaumburg, IL, April 18-20, 1994, ASM International, Indianapolis, IN, pp. $455-461$.

[22] Vanpaemel, J., "History of the Hardening of Steel : Science and Technology," J. Phys. Colloques, Vol. 43, No. 4, 1982, pp. 847-854.

[23] MacKenzie, D. S. and Graham, G., "Beer, Blood and Urine-Mythological Quenchants of Ancient Blacksmiths," presented at the 23rd IFHTSE Congress, Savannah, GA, April 18-21, 2016, International Federation of Heat Treatment and Surface Engineering, Savannah, GA, pp. 101-109.

[24] ISO 9950, Industrial Quenching Oils-determination of Cooling Characteristics-Nickelalloy Probe Test Method, International Organization for Standardization, Geneva, Switzerland, 1995, www.iso.org 
[25] ASTM D6200-01, Standard Test Method for Determination of Cooling Characteristics of Quench Oils by Cooling Curve Analysis, ASTM International, West Conshohocken, PA, 2017, www.astm.org

[26] ASTM D6482-06, Standard Test Method for Determination of Cooling Characteristics of Aqueous Polymer Quenchants by Cooling Curve Analysis with Agitation (Tensi Method), ASTM International, West Conshohocken, PA, 2016, www.astm.org

[27] ASTM D6549-06, Standard Test Method for Determination of Cooling Characteristics of Quenchants by Cooling Curve Analysis with Agitation (Drayton Unit), ASTM International, West Conshohocken, PA, 2015, www.astm.org

[28] JB/T 4392, Method for Determination of Properties of Water-Soluble Quenching Medium for Organic Compounds (in Chinese), GB Standards, Shenzhen, China, 1999, www.gbstandards.org

[29] JB/T 7951-2004, Industrial Quenching Oil. Determination of Cooling Characteristics. Nickel-Alloy Probe Test Method (in Chinese), GB Standards, Shenzhen, China, 2004, www.gbstandards.org

[30] Totten, G. E., Bates, C. E., and Clindon, N. A., Handbook of Quenchants and Quenching Technology, ASM International, Materials Park, OH, 1993, 507p.

[31] Roberts, G. A., Hamaker, J. C., and Johnson, A. R., Tool Steels, 3rd ed., ASM International, Materials Park, OH, 1962, 315p.

[32] Liščić, B., "Possibilities of Heat Transfer Control During Quenching," Mater. Technol., Vol. 42, No. 11, 2008, pp. 1-19.

[33] Luo, X. M. and Li, J. K., "Dynamic Characteristics upon Quench-Cooling and Actual Quenching of Steel Workpieces (in Chinese)," Heat Treat., Vol. 24, No. 5, 2009, pp. 44-49.

[34] Liščić, B., Tens, H. M., and Lauralice, C. F., Quenching Theory and Technology, 2nd ed., CRC Press, Boca Raton, FL, 2010, 725p.

[35] Bobok, A. N., "Use of Fluidized Bed in Heat Treatment Shops of the Machine Building Industry under Conditions of the Competitive Market," Met. Sci. Heat Treat., Vol. 47, Nos. 3-4, 2005, pp. 97-102.

[36] Liščić, B, Tensi, H. M., and Luty, W., Theory and Technology of Quenching, SpringerVerlag Berlin, Heidelberg, Germany, 1992, 484p.

[37] Totten, G. E., Luigi, L. M., Albano, L. L. M., and Lauralice, C. F. C., "Into the 'Black Hole' of Heat Treating: Brine Quenching," presented at the Fifth International Conference on Heat Treatment and Surface Engineering in Asia, Hangzhou, China, Nov. 12-14, 2016, Chinese Heat Treatment Society, Beijing, China, pp. 18-29.

[38] Liščić, B., "Measurement and Recording of Quenching Intensity in Workshop Conditions Based on Temperature Gradients," Mater. Perform. Charact., Vol. 5, No. 1, 2016, pp. 209-226, https://doi.org/10.1520/MPC20160007

[39] Kobasko, N. and Liščić, B., "Liščić/Petrofer Probe to Investigate Real Industrial Hardening Processes and Some Fundamentals of Quenching Steel Parts in Liquid Media," Phys. Eng., Vol. 6, No. 6, 2017, pp. 48-56.

[40] JIS K 2242, Heat Treating Fluids, Japanese Industrial Standard / Japanese Standards Association, Tokyo, Japan, 2012, www.jsa.or

[41] Funatani, K., Narazaki, M., and Tanaka, M., "Evaluation of Testing Methods of Cooling Power of Liquid Quenchants," presented at the Third International Conference on Quenching and Control of Distortion, Prague, Czech Republic, March 14-26, 1999, ASM International, Materials Park, OH, pp. 515-521.

[42] ASTM D7646-10, Standard Test Method for Determination of Cooling Characteristics of Aqueous Polymer Quenchants for Aluminum Alloys by Cooling Curve Analysis, ASTM International, West Conshohocken, PA, 2014, www.astm.org

[43] Segerberg, S. O., "Classification of Quench Oils: A Method of Comparison," Heat Treat., Vol. 20, No. 12, 1988, pp. 30-33.

[44] Zeng, G. Y., "Identification and Evaluation for Cooling Curve of Quenchants," Met. Heat Treat., Vol. 3, 1999, pp. 38-40.

[45] Chen, C. H. and Zhou, J. E., "Analysis of the Segerberg Hardening Power Equation," Quenching and Cooling, Residual Stress and Distortion Control, ASTM STP1523, 
C. Lauralice and N. Michiharu, Eds., ASTM International West Conshohocken, PA, 2010, 11p, https://doi.org/10.1520/STP49195S

[46] Luo, X. M. and Totten, G. E., "Distortion Control: Quenching Apparatus for Hardening Parts: An Overview," Mater. Perform. Charact., Vol. 3, No. 4, 2014, pp. 1-28, https://doi.org/10.1520/MPC20130086

[47] Li, F., "Computer Simulation of Quenchant Flow-Field in Quenching Tank with Agitating Unit (in Chinese)," M.A. thesis, Jiangsu University, Jiangsu, China, 2005.

[48] Kumar, A., Metwally, H., Paingankar, S., and MacKenzie, D. S., "Evaluation of Flow Uniformity around Automotive Pinion Gears during Quenching," Härtereitechnische Mitteilungen, Vol. 62, No. 6, 2007, pp. 274-278, https://doi.org/10.3139/105.100438

[49] Chen, N., Zhang, W. M., Li, Q., Gao, C. Y., Liao, B., and Pan, J. S., "Optimization of Quench Tank Structure Based on CFD Simulation,” Solid State Phenom., Vol. 118, 2006, pp. 363-368, https://doi.org/10.4028/www.scientific.net/SSP.118.363

[50] Bineli, A. R. R., Barbosa, M. I. R., Jardini, A. L., and Filho, R. M., "Simulation to Analyze Two Models of Agitation System in Quench Process," Comput. Aided Chem. Eng., Vol. 25, 2010, pp. 1715-1720.

[51] Banka, A., Franklin, J., Li, Z., Lynn, F. B., and Aronov, M., "CFD and FEA Used to Improve the Quenching Process," Heat Treat. Prog., 2008, pp. 50-56.

[52] Krukovskyi, P., Kobasko, N., and Yurchenko, D., "Generalized Equation for Cooling Time Evaluation and Its Verification by CFD Analysis," J. ASTM Int., Vol. 6, No. 5, 2011, pp. 1-15, https://doi.org/10.1520/JAI101760

[53] MacKenzie, D. S., Kumar, A., and Metwally, H., "Optimizing Agitation and Quench Uniformity Using CFD," presented at Proceedings of the 23rd ASM Heat Treating Society Conference, Pittsburgh, PA, Sep. 25-28, 2005, ASM International, Materials Park, OH, pp. 271-278.

[54] Kobasko, N. I., Aronov, M. A., and Powell, J. A., Intensive Quenching System: Engineering and Design, ASTM International, West Conshohocken, PA, 2010, 252p.

[55] Aronov, M. A., Final Technical Report: Intensive Quenching Technology for Heat Treating and Forging Industries, DOE Award Number: DE-FC36-03ID, EMTEC, Dayton, OH, 2005, 50p.

[56] Aronov, M. A., Kobasko, N. I., and Powell, J. A., "Industrial-Scale Intensive Quenching Process for Tool Products Commercialization," Report No. EMTEC CT-76-II, EMTEC, Dayton, OH, 2005, 28p.

[57] Kobasko, N. I., "Steel Superstrengthening Phenomenon, Part I," Int. J. Mater. Prod. Technol., Vol. 24, Nos. 1-4, 2005, pp. 361-374.

[58] Totten, G. E., Steel Heat Treatment: Equipment and Process Design, Taylor \& Francis, New York, NY, 2006, 714p.

[59] Dossett, J. I. and Totten, G. E, ASM Handbook, Vol. 4A: Steel Heat Treating Fundamentals and Processes, ASM International, Materials Park, OH, 2013, 784p.

[60] Chen, N. L. and Zhang, W. M., "Application of Numerical Control Technology of Quench-Cooling (in Chinese)," presented at the Ninth National Heat Treatment Conference, Dailan, China, Sep. 16-19, 2007, Chinese Heat Treatment Society, Dalian, China, pp. 90-96.

[61] Totten, G. E., Steel Heat Treatment: Equipment and Process Design, CRC press, Taylor \& Francis, New York, NY, 2007, 714p. 